\title{
ON CHIRAL DIFFERENTIAL OPERATORS OVER HOMOGENEOUS SPACES
}

\author{
VASSILY GORBOUNOV, FYODOR MALIKOV, and VADIM SCHECHTMAN
}

(Received 27 August 2000 and in revised form 15 February 2001)

\begin{abstract}
We give a classification and construction of chiral algebras of differential operators over semisimple algebraic groups $G$ and over homogeneous spaces $G / N$ and $G / P$ where $N$ is a nilpotent and $P$ a parabolic subgroup.
\end{abstract}

2000 Mathematics Subject Classification. 14Lxx, 22E67, 14-XX.

1. Introduction. The notion of an algebra of chiral differential operators (cdo for short) over a smooth algebraic variety $X$ has been studied in [8]. (This notion has been invented and first studied, in a different language, by Beilinson and Drinfeld, [1, Chapter 3, Section 8].) In the present paper, we consider some examples in more details. We will work over the ground field $\mathbb{C}$.

We give a classification of cdo over $X$ in the following cases: $X=G$ is an affine algebraic group; $X=G / N$ or $G / P$, where $N$ is a unipotent subgroup and $P$ is a parabolic subgroup and $G$ is simple (the extension to the case of a semi-simple $G$ being straightforward).

Before we describe the result, we explain some terminologies and notations. For a smooth algebraic variety $X$, an algebra of cdo over $X$ is by definition a Zariski sheaf of of $\mathbb{Z}_{\geq 0}$-graded vertex algebras on $X$ such that

(a) if $\mathscr{A} \lg (\mathscr{V})=(\mathscr{A}, \mathscr{T}, \Omega, \partial, \gamma,\langle\cdot, \cdot\rangle, c)$ is the sheaf of vertex algebroids associated with $\mathscr{V}$ (see [8, Section 2]), then the corresponding extended Lie algebroid $(\mathscr{A}, \mathcal{T}, \Omega, \partial$ ) (see (2.1)) is identified with $\left(O_{X}, \Theta_{X}, \Omega_{X}^{1}, d_{\mathrm{DR}}\right)$, where $\Theta_{X}$ denotes the tangent bundle and $d_{\mathrm{DR}}$ the de Rham differential;

(b) the adjunction morphism $U \mathscr{A l g}(\mathscr{V}) \rightarrow \mathscr{V}$ is an isomorphism. Here $U$ is the functor of vertex envelope defined in [8, Section 9]

For more details see Section 2.

For each Zariski open $U \subset X$, we can consider the category (a groupoid in fact) of cdo over $U$, or, what is the same, the groupoid of vertex algebroids (defined in [8, Section 3]) over $U$ satisfying (a) above. When $U$ varies, we get a sheaf of groupoids Diff ${ }_{X}^{\text {ch }}$ over $X$-the gerbe of chiral differential operators. As usual, $\Gamma\left(U\right.$; $\left.\mathscr{D i f f}{ }_{X}^{\mathrm{ch}}\right)$ denote the sections over $U$; a generic object of this category is sometimes denoted by $\mathscr{D}_{U}^{\mathrm{ch}}$; the set of isomorphism classes of cdo over $U$ is denoted by $\pi_{0}\left(\Gamma\left(U ; \mathscr{D i f f} f_{X}^{\mathrm{ch}}\right)\right)$.

Let $G$ be an affine connected algebraic group, and let $\mathfrak{g}$ be the corresponding Lie algebra. For each symmetric ad-invariant bilinear form $(\cdot, \cdot) \in\left(S^{2} \mathfrak{g}^{*}\right)^{\mathfrak{g}}$ we construct a cdo $\mathscr{D}_{G ;(\cdot, \cdot)}^{\mathrm{ch}}$ over $G$ such that if $G$ is semi-simple, then the correspondence $(\cdot, \cdot) \mapsto$ $\mathscr{D}_{G ;(\cdot,)}^{\mathrm{ch}}$ gives rise to a bijection 


$$
\left(S^{2} \mathfrak{g}^{*}\right)^{\mathfrak{g}} \stackrel{\sim}{\longrightarrow} \pi_{0}\left(\Gamma\left(G, \text { Diff }_{G}^{\mathrm{ch}}\right)\right) .
$$

We have a canonical embedding of vertex algebras

$$
i_{(\cdot, \cdot)}: \mathscr{V}_{\mathfrak{g},(\cdot, \cdot)} \longleftrightarrow \mathscr{D}_{G ;(\cdot, \cdot)}^{\mathrm{ch}},
$$

where $\mathscr{V}_{\mathfrak{g} ;(\cdot, \cdot)}$ denotes the vacuum module of the Kac-Moody algebra $\hat{\mathfrak{g}}$ at level $(\cdot, \cdot)$. This embedding is induced by the embedding of $\mathfrak{g}$ into $T_{G}:=\Gamma\left(G ; \Theta_{G}\right)$ as left invariant vector fields.

Let $(\cdot, \cdot)_{\mathfrak{g} ;(K)}$ denote the Killing form on $\mathfrak{g}$. We define the dual level by

$$
(\cdot, \cdot)^{o}=-(\cdot, \cdot)_{\mathfrak{g} ;(K)}-(\cdot, \cdot) .
$$

Using the embedding $\mathfrak{g} \hookrightarrow T_{G}$ by means of right invariant vector fields, one can construct a canonical dual embedding of vertex algebras

$$
i_{(\cdot, \cdot)}^{o}: \mathscr{V}_{\mathfrak{g} ;(\cdot, \cdot)^{o}} \longrightarrow \mathscr{D}_{G ;(\cdot, \cdot)}^{\mathrm{ch}} \cdot
$$

It is characterised by the requirement that the images of $i_{(\cdot, \cdot)}$ and $i_{(\cdot, \cdot)}^{o}$ commute in an appropriate sense (see Theorem 4.1 and Corollary 4.2). This beautiful fact was communicated to us by B. Feigin, E. Frenkel, and D. Gaitsgory. We give a proof using the language of [8].

We pass to homogeneous spaces. Assume that $G$ is simple. Let $N \subset G$ be a unipotent group. The classification of cdo over $G / N$ is the same as over $G$; namely, for each level $(\cdot, \cdot)$ one can define a cdo $\mathscr{D}_{G / N ;(\cdot, \cdot)}^{\mathrm{ch}}$ such that the correspondence $(\cdot, \cdot) \mapsto \mathscr{D}_{G / N ;(\cdot, \cdot)}^{\mathrm{ch}}$ induces a bijection

$$
\left(S^{2} \mathfrak{g}^{*}\right)^{\mathfrak{g}} \stackrel{\sim}{\longrightarrow} \pi_{0}\left(\Gamma\left(\frac{G}{N} ; \mathscr{D i f f} \mathrm{Gh}_{/ N}^{\mathrm{c}}\right)\right) .
$$

The sheaves $\mathscr{D}_{G / N ;(\cdot, \cdot)}^{\mathrm{ch}}$ are constructed using the BRST (or quantum Hamiltonian) reduction of the corresponding cdo's on $G$. More precisely,

$$
\mathscr{D}_{G / N ;(\cdot, \cdot)}^{\mathrm{ch}}=H_{\mathrm{BRST}}^{0}\left(L \mathfrak{n} ; \pi_{*} \mathscr{D}_{G ;(\cdot, \cdot)}^{\mathrm{ch}}\right),
$$

where the right-hand side denotes the BRST cohomology of the loop algebra $L \mathfrak{n}:=$ $\mathfrak{n}\left[T, T^{-1}\right], \mathfrak{n}:=\operatorname{Lie}(N)$. For the precise definition see Section 5.

Let $B \subset G$ be a Borel subgroup. We show that there exists a unique, up to a unique isomorphism, cdo $\mathscr{D}_{G / B}^{\mathrm{ch}}$ on the flag space $G / B$. Again this cdo may be constructed using the BRST reduction. Namely,

$$
\mathscr{D}_{G / B}^{\mathrm{ch}}=H_{\mathrm{BRST}}^{0}\left(L \mathfrak{b}, \mathfrak{h} ; \pi_{*} \mathscr{D}_{G ; \mathrm{crit}}^{\mathrm{ch}}\right) .
$$

Here $\mathscr{D}_{G ; \text {;rit }}^{\text {ch }}$ is, by definition, the cdo $\mathscr{D}_{G ;(\cdot, \cdot))_{\text {crit }}}^{\text {ch }}$ on the critical level $(\cdot, \cdot)_{\text {crit }}=-(\cdot, \cdot)_{\mathfrak{g} ;(K)} / 2$. For the definition of the relative BRST cohomology in the right-hand side, we again refer the reader to the main body of the paper, see Section 6. (A more explicit construction of the sheaf $\mathscr{D}_{G / B}^{\mathrm{ch}}$ for $G=\mathrm{SL}(n)$, using vertex operators, has been suggested in $[11,5.9,5.10]$.

The embeddings (1.4) induce canonical morphisms of vertex algebras

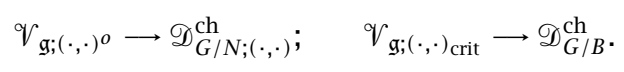


Taking the spaces of sections over a big cell, we get another construction of FeiginFrenkel Wakimoto modules (cf. [5, 6, 7]).

Finally, if $P \subset G$ is parabolic but not Borel, we show that $\Gamma\left(G / P ; \mathscr{D i f f} \mathrm{ch}_{P}^{\mathrm{ch}}\right)$ is empty. The classification of cdo over homogeneous spaces is exactly reflected in the BRST world, namely, the square of the corresponding BRST charge is zero at all levels for $G / N$, only at the critical level for $G / B$ and is never zero for $G / P$.

This introduction would not be complete without mentioning that this paper relies heavily on the ideas of B. Feigin and E. Frenkel. This paper started from our attempts to find a proof of Theorem 4.1 and Corollary 4.2. Our sincere gratitude goes to D. Gaitsgory who had communicated these facts to us and told us that he had known their proofs. We are also grateful to H. Esnault for a crucial Remark 6.1.

2. Preliminaries on cdo. In this section, we recall the necessary definitions and theorems from [8]; for all the details the reader is referred to the references therein.

2.1. Let $A$ be a commutative $\mathbb{C}$-algebra. We denote by $T_{A}$ the Lie algebra of $\mathbb{C}$ derivations $\operatorname{Der}_{\mathbb{C}}(A, A)$; it is also canonically a left $A$-module. Let $\Omega_{A}$ denote the $A$ module of Kähler differentials $\Omega_{A / \mathbb{C}}^{1}=\operatorname{Hom}_{A}\left(T_{A}, A\right)$ and $d: A \rightarrow \Omega_{A}$ the de Rham differential. The Lie algebra $T_{A}$ acts canonically on $\Omega_{A}$ and $d$ commutes with the action of $T_{A}$. We have the tautological $A$-bilinear pairing $\langle\cdot, \cdot\rangle: T_{A} \times \Omega_{A} \rightarrow A$, and all these structures satisfy the standard identities if differential geometry, cf. [8, (0.1.3)-(0.1.6)] and (2.2), (2.3), (2.4), and (2.5) below.

A Lie $A$-algebroid is a Lie algebra $T$ acting by derivations on $A$ and equipped with a structure of a left $A$-module, such that

$$
[\tau, a v]=a[\tau, v]+\tau(a) v, \quad(a \tau)(b)=a \tau(b)
$$

for all $\tau, v \in T ; a, b \in A$. For example, $T_{A}$ is a Lie $A$-algebroid.

2.2. An extended Lie algebroid is a quintuple $\mathscr{T}=(A, T, \Omega, \partial,\langle\cdot, \cdot\rangle)$, where $A$ is as above, $T$ is a Lie $A$-algebroid, $\Omega$ is an $A$-module equipped with a structure of a module over the Lie algebra $T, \partial: A \rightarrow \Omega$ is an $A$-derivation and a morphism of $T$-modules, $\langle\cdot, \cdot\rangle: T \times \Omega \rightarrow A$ is an $A$-bilinear pairing.

These data must satisfy the following properties $(a \in A, \tau, v \in T, \omega \in \Omega)$ :

$$
\begin{gathered}
\langle\tau, \partial a\rangle=\tau(a), \\
\tau(a \omega)=\tau(a) \omega+a \tau(\omega), \\
(a \tau)(\omega)=a \tau(\omega)+\langle\tau, \omega\rangle \partial a, \\
\tau(\langle\nu, \omega\rangle)=\langle[\tau, v], \omega\rangle+\langle v, \tau(\omega)\rangle .
\end{gathered}
$$

We also say that $\mathcal{T}=(A, T, \ldots)$ is an extended Lie $A$-algebroid.

We say that $\mathscr{T}$ is perfect if the pairing $\langle\cdot, \cdot\rangle$ induces an isomorphism $\Omega \stackrel{\sim}{\longrightarrow}$ $\operatorname{Hom}_{A}(T, A)$. Given a Lie $A$-algebroid $T$, we may set $\Omega:=\operatorname{Hom}_{A}(T, A)$, define $\partial$ by (2.2) and the $T$-action on $\Omega$ by (2.5); this way we get a perfect extended Lie algebroid.

2.3. A vertex algebroid is a septuple $A=(A, T, \Omega, \partial, \gamma,\langle\cdot, \cdot\rangle, c)$, where $A$ is as above, $T$ is a Lie $A$-algebroid, $\Omega$ is an $A$-module equipped with an action of the Lie algebra 
$T, \partial: A \rightarrow \Omega$ is a derivation commuting with the $T$-action,

$$
\langle\cdot, \cdot\rangle:(T \oplus \Omega) \times(T \oplus \Omega) \longrightarrow A
$$

is a symmetric $\mathbb{C}$-bilinear pairing equal to zero on $\Omega \times \Omega$ and such that $\mathscr{T}_{\mathscr{A}}=$ $\left(A, T, \Omega, \partial,\left.\langle\cdot, \cdot\rangle\right|_{T \times \Omega}\right)$ is an extended Lie $A$-algebroid; $c: T \times T \rightarrow \Omega$ is a skew symmetric $\mathbb{C}$-bilinear pairing and $\gamma: A \times T \rightarrow \Omega$ is a $\mathbb{C}$-bilinear map.

The following axioms must hold $\left(a, b \in A ; \tau, \tau_{i} \in T\right)$ :

$$
\begin{gathered}
\gamma(a, b \tau)=\gamma(a b, \tau)-a \gamma(b, \tau)-\tau(a) \partial b-\tau(b) \partial a, \\
\left\langle a \tau_{1}, \tau_{2}\right\rangle=a\left\langle\tau_{1}, \tau_{2}\right\rangle+\left\langle\gamma\left(a, \tau_{1}\right), \tau_{2}\right\rangle-\tau_{1} \tau_{2}(a), \\
c\left(a \tau_{1}, \tau_{2}\right)=a c\left(\tau_{1}, \tau_{2}\right)+\gamma\left(a,\left[\tau_{1}, \tau_{2}\right]\right)-\gamma\left(\tau_{2}(a), \tau_{1}\right)+\tau_{2}\left(\gamma\left(a, \tau_{1}\right)\right) \\
-\frac{1}{2}\left\langle\tau_{1}, \tau_{2}\right\rangle \partial a+\frac{1}{2} \partial \tau_{1} \tau_{2}(a)-\frac{1}{2} \partial\left\langle\tau_{2}, \gamma\left(a, \tau_{1}\right)\right\rangle, \\
\left\langle\left[\tau_{1}, \tau_{2}\right], \tau_{3}\right\rangle+\left\langle\tau_{2},\left[\tau_{1}, \tau_{3}\right]\right\rangle=\tau_{1}\left(\left\langle\tau_{2}, \tau_{3}\right\rangle\right)-\frac{1}{2} \tau_{2}\left(\left\langle\tau_{1}, \tau_{3}\right\rangle\right)-\frac{1}{2} \tau_{3}\left(\left\langle\tau_{1}, \tau_{2}\right\rangle\right) \\
+\left\langle\tau_{2}, c\left(\tau_{1}, \tau_{3}\right)\right\rangle+\left\langle\tau_{3}, c\left(\tau_{1}, \tau_{2}\right)\right\rangle, \\
d_{\text {Lie }} c\left(\tau_{1}, \tau_{2}, \tau_{3}\right)=-\frac{1}{2} \partial\left\{\left\langle\left[\tau_{1}, \tau_{2}\right], \tau_{3}\right\rangle+\left\langle\left[\tau_{1}, \tau_{3}\right], \tau_{2}\right\rangle-\left\langle\left[\tau_{2}, \tau_{3}\right], \tau_{1}\right\rangle\right. \\
\left.-\tau_{1}\left(\left\langle\tau_{2}, \tau_{3}\right\rangle\right)+\tau_{2}\left(\left\langle\tau_{1}, \tau_{3}\right\rangle\right)-2\left\langle\tau_{3}, c\left(\tau_{1}, \tau_{2}\right)\right\rangle\right\},
\end{gathered}
$$

where

$$
\begin{aligned}
d_{\mathrm{Lie}} c\left(\boldsymbol{\tau}_{1}, \boldsymbol{\tau}_{2}, \boldsymbol{\tau}_{3}\right)= & -c\left(\left[\boldsymbol{\tau}_{1}, \boldsymbol{\tau}_{2}\right], \boldsymbol{\tau}_{3}\right)+c\left(\left[\boldsymbol{\tau}_{1}, \boldsymbol{\tau}_{3}\right], \boldsymbol{\tau}_{2}\right)-c\left(\left[\boldsymbol{\tau}_{2}, \boldsymbol{\tau}_{3}\right], \boldsymbol{\tau}_{1}\right) \\
& +\boldsymbol{\tau}_{1}\left(c\left(\boldsymbol{\tau}_{2}, \boldsymbol{\tau}_{3}\right)\right)-\boldsymbol{\tau}_{2}\left(c\left(\boldsymbol{\tau}_{1}, \boldsymbol{\tau}_{3}\right)\right)+\boldsymbol{\tau}_{3}\left(c\left(\boldsymbol{\tau}_{1}, \boldsymbol{\tau}_{2}\right)\right) .
\end{aligned}
$$

We call $\mathscr{A}$ perfect if the corresponding extended Lie algebroid $\mathscr{T}_{\mathscr{A}}$ is perfect.

2.4. Pushout. Let $\mathscr{A}=(A, T, \Omega, \ldots)$ be a vertex $A$-algebroid. Let $B$ be a commutative $A$-algebra, and let $i: A \rightarrow B$ be the structure morphism. Set $\Omega_{B}:=B \otimes_{A} \Omega, T_{B}:=B \otimes T$. The $A$-derivation $\partial: A \rightarrow \Omega$ induces a $B$-derivation $\partial_{B}: B \rightarrow \Omega_{B}$. The $A$-bilinear pairing $\langle\cdot, \cdot\rangle: T \times \Omega \rightarrow A$ uniquely extends to a $B$-bilinear pairing $\langle\cdot, \cdot\rangle_{B}: T_{B} \times \Omega_{B} \rightarrow B$.

Assume that the Lie algebra $T$ acts on $B$ by derivations in such a way that $T(i(a))=$ $i(\tau(a))$ and $(a \tau)(b)=a \tau(b)(a \in A, b \in B, \tau \in T)$. Then $T_{B}$ acquires a canonical structure of a Lie $B$-algebroid. Namely, the Lie bracket on $T_{B}$ is given by

$$
\left[b_{1} \otimes \tau_{1}, b_{2} \otimes \tau_{2}\right]=b_{1} b_{2} \otimes\left[\tau_{1}, \tau_{2}\right]+\tau_{1}\left(b_{2}\right) b_{1} \otimes \tau_{2}-\tau_{2}\left(b_{1}\right) b_{2} \otimes \tau_{1},
$$

and the action of $T_{B}$ on $B$ is defined by

$$
\left(b_{1} \otimes \tau\right)\left(b_{2}\right)=b_{1} \tau\left(b_{2}\right) .
$$

In such a way, $\left(T_{B}, \Omega_{B}, \partial_{B},\langle\cdot, \cdot\rangle_{B}\right)$ becomes an extended Lie $B$-algebroid.

THEOREM 2.1 (cf. [8, Theorem 1.10.1]). Assume that we are given a $\mathbb{C}$-bilinear mapping $\gamma: B \times T \rightarrow \Omega_{B}$ such that $\gamma(i(a), \tau)=1 \otimes \gamma(a, \tau)$ and that (2.7) holds true for all $\tau \in T, a \in B, b \in A$. 
Then there exists a unique extension of $\gamma$ to a $\mathbb{C}$-bilinear mapping $\gamma_{B}: B \times T_{B} \rightarrow \Omega_{B}$ satisfying (2.7) for all $a, b \in B, \tau \in T_{B}$; there exists a unique extension of the pairing $\langle\cdot, \cdot\rangle: T \times T \rightarrow A$ to a pairing $\langle\cdot, \cdot\rangle_{B}: T_{B} \times T_{B} \rightarrow B$ satisfying (2.8) for all $a \in B, \tau_{i} \in T_{B}$; there exists a unique extension of the pairing $c: T \times T \rightarrow \Omega$ to a pairing $c_{B}: T_{B} \times T_{B} \rightarrow \Omega_{B}$ satisfying (2.9) for all $a \in B, \tau_{i} \in T_{B}$.

The septuple $\mathscr{A}_{B}=\left(B, T_{B}, \Omega_{B}, \partial_{B}, \gamma_{B},\langle\cdot, \cdot\rangle_{B}, C_{B}\right)$ is a vertex $B$-algebroid.

2.5. Morphisms. Let $\mathscr{A}=(A, T, \Omega, \ldots)$ and $\mathscr{A}^{\prime}=\left(A^{\prime}, T^{\prime}, \Omega^{\prime}, \ldots\right)$ be two vertex algebroids. A morphism $g: \mathscr{A} \rightarrow \mathscr{A}^{\prime}$ is, by definition, a quadruple $g=\left(g_{A}, g_{T}, g_{\Omega}, h\right)$, where

(1) $g_{A}: A \rightarrow A^{\prime}$ is a morphism of $\mathbb{C}$-algebras;

(2) $g_{\Omega}: \Omega \rightarrow \Omega^{\prime}$ is a morphism of $k$-modules such that

$$
g_{\Omega}(\partial a)=\partial g_{A}(a), \quad g_{\Omega}(a \omega)=g_{A}(a) g_{\Omega}(\omega)
$$

(3) $g_{T}: T \rightarrow T^{\prime}$ is a morphism of Lie $k$-algebras such that

$$
\begin{gathered}
g_{T}(a \tau)=g_{A}(a) g_{T}(\tau), \quad g_{A}(\tau(a))=g_{T}(\tau)\left(g_{A}(a)\right), \\
g_{\Omega}(\tau(\omega))=g_{T}(\tau)\left(g_{\Omega}(\omega)\right), \quad g_{A}(\langle\tau, \omega\rangle)=\left\langle g_{T}(\tau), g_{\Omega}(\omega)\right\rangle ;
\end{gathered}
$$

(4) $h: T \rightarrow \Omega^{\prime}$ is a morphism of $k$-modules such that

$$
h(a \tau)=g_{A}(a) h(\tau)-\gamma^{\prime}\left(g_{A}(a), g_{T}(\tau)\right)+g_{\Omega}(\gamma(a, \tau)) ;
$$

$$
\begin{aligned}
g_{A}\left(\left\langle\tau_{1}, \tau_{2}\right\rangle\right)= & \left\langle g_{T}\left(\tau_{1}\right), g_{T}\left(\tau_{2}\right)\right\rangle^{\prime}+\left\langle g_{T}\left(\tau_{1}\right), h\left(\tau_{2}\right)\right\rangle+\left\langle g_{T}\left(\tau_{2}\right), h\left(\tau_{1}\right)\right\rangle \\
g_{\Omega}\left(c\left(\tau_{1}, \tau_{2}\right)\right)= & c^{\prime}\left(g_{T}\left(\tau_{1}\right), g_{T}\left(\tau_{2}\right)\right)+\frac{1}{2} \partial\left\langle g_{T}\left(\tau_{1}\right), h\left(\tau_{2}\right)\right\rangle-\frac{1}{2} \partial\left\langle g_{T}\left(\tau_{2}\right), h\left(\tau_{1}\right)\right\rangle \\
& -g_{T}\left(\tau_{1}\right)\left(h\left(\tau_{2}\right)\right)+g_{T}\left(\tau_{2}\right)\left(h\left(\tau_{1}\right)\right)+h\left(\left[\tau_{1}, \tau_{2}\right]\right),
\end{aligned}
$$

(cf. [8, Theorem 3.5]).

If $g^{\prime}: \mathscr{A}^{\prime} \rightarrow \mathscr{A}^{\prime \prime}$ is another morphism, then the composition $g^{\prime} \circ g$ is defined by

$$
\mathfrak{g}^{\prime} \circ \mathfrak{g}=\left(\mathfrak{g}_{A}^{\prime} \mathfrak{g}_{A}, \mathfrak{g}_{T}^{\prime} \mathfrak{g}_{T}, g_{\Omega}^{\prime} g_{\Omega}, g_{\Omega}^{\prime} h+h^{\prime} g_{T}\right)
$$

The identity morphisms are

$$
\operatorname{Id}_{\mathscr{A}}=\left(\operatorname{Id}_{A}, \operatorname{Id}_{T}, \operatorname{Id}_{\Omega}, 0\right) .
$$

This way we get a category of vertex algebroids Alg.

2.6. Let $V$ be a $\mathbb{Z}_{\geq 0}$-graded vertex algebra. Thus, $V$ is a $\mathbb{Z}_{\geq 0}$-graded vector space $V=\oplus_{i \geq 0} V_{i}$ equipped with an endomorphism $\partial: V \rightarrow V$ of degree 1 , a vector $\mathbf{1} \in V_{0}$ and an infinite set of binary operations ${ }_{(n)}: V \otimes V \rightarrow V$ of degree $-n-1(n \in \mathbb{Z})$. These data must satisfy the axioms listed, for example, in [8, 0.5].

Set $A=V_{0}$; let $\Omega \subset V_{1}$ be the subspace generated by all elements $a_{(-1)} \partial b(a, b \in A)$; set $T=V_{1} / \Omega$. We choose a splitting

$$
s: T \rightarrow V_{1}
$$


of the projection $V_{1} \rightarrow T$. Using operations $(n), n=-1,0,1$, one defines a vertex algebroid $\mathscr{A}(V)=(A, T, \Omega, \partial, \gamma,\langle\cdot, \cdot\rangle, c)$. Roughly speaking, the multiplication on $A$ and the structures of $A$-modules on $T$ and $\Omega$ are induced by (-1), the Lie bracket on $T$ and the action of $T$ on $A$ and $\Omega$ are induced by $(0)$, and the pairing $\langle\cdot, \cdot\rangle$ is induced by (1). For the details, see Section 2.

This algebroid depends on the choice of a splitting $s$, (2.22), so the notation for it could be $\mathscr{A}(V, s)$. However, given the second splitting $s^{\prime}$, there exists a canonical isomorphism of vertex algebroids $\phi_{s, s^{\prime}}: \mathscr{A}\left(V, s^{\prime}\right) \stackrel{\sim}{\longrightarrow} \mathscr{A}(V, s)$. We have $\phi_{s, s}=\operatorname{Id}_{\mathscr{A}(V, s)}$ and $\phi_{s, s^{\prime}} \circ \phi_{s^{\prime}, s^{\prime \prime}}=\phi_{s, s^{\prime \prime}}$. This way we get a functor

$$
A: \mathscr{V} \text { ert } \rightarrow A l g
$$

from category of $\mathbb{Z}_{\geq 0}$-graded vertex algebras to the category of vertex algebroids.

This functor admits a left adjoint

$$
U: A \lg \longrightarrow \mathscr{V e r t}
$$

called vertex envelope, see [8, Section 9]. This functor is fully faithful; for each $\mathscr{A}$ the adjunction morphism $\mathscr{A} \rightarrow \mathscr{A}(U \mathscr{A})$ is an isomorphism.

2.7. We call a vertex algebroid $\mathscr{A}=(A, T, \ldots)$ standard if it is perfect and the canonical morphism $T \rightarrow T_{A}=\operatorname{Der}_{\mathbb{C}}(A, A)$ is an isomorphism.

We fix an algebra $A$ and consider the subcategory $\mathscr{A} \lg _{A} \subset A \lg$ whose objects are standard algebroids whose underlying commutative algebra is $A$, and whose morphisms are quadruples $g=\left(g_{A}, g_{T}, g_{\Omega}, h\right)$ with $g_{A}=\operatorname{Id}_{A}$. This category is a groupoid, that is, all morphisms are isomorphisms.

We define a category $\Omega_{A}^{[2,3\rangle}$ whose objects are closed 3-forms $\omega \in \Omega_{A}^{3, c l}$, a morphism between $\omega$ and $\omega^{\prime}$ being a 2 -form $\eta \in \Omega_{A}^{2}$ such that $d \eta=\omega^{\prime}-\omega$. The composition of morphisms is given by the addition of 2 -forms. The addition of 3-forms makes $\Omega_{A}^{[2,3\rangle}$ an Abelian group in categories.

If $\mathscr{A}=(A, T, \Omega, \partial, \gamma,\langle\cdot, \cdot\rangle, c) \in \mathscr{A} \lg _{A}$ and $\omega \in \Omega_{A}^{3, \mathrm{cl}}$, then

$$
\mathscr{A}+\omega:=(A, T, \Omega, \partial, \gamma,\langle\cdot, \cdot\rangle, c+\omega)
$$

is also an object of $\mathscr{A} \lg _{A}$. This way we get an action

$$
+: A \lg _{A} \times \Omega_{A}^{[2,3\rangle} \longrightarrow \mathscr{A} \lg _{A}
$$

which makes $\mathscr{A} \lg _{A}$ an $\Omega_{A}^{[2,3\rangle}$-torseur. This means that, for each $\mathscr{A} \in \mathscr{A} \lg _{A}$ the functor $\Omega_{A}^{[2,3\rangle} \rightarrow \mathscr{A} \lg _{A}$ sending $\omega$ to $\mathscr{A}+\omega$ is an equivalence of categories, see [8, Example 7.1].

If $T_{A}$ is a free $A$-module admitting a base which consists of mutually commuting vector fields then $A \lg _{A}$ is nonempty, see [8, Example 7.1].

A vertex algebra $V$ is called a cdo over $A$ if $\mathscr{A}(V) \in \mathscr{A} \lg _{A}$ and the adjunction morphism $U \mathscr{A}(V) \rightarrow V$ is an isomorphism. Such algebras form a category $\mathscr{C D O} \mathscr{O}_{A}$ and the functor of vertex envelope

$$
U: A \lg _{A} \longrightarrow \mathscr{C D O}_{A}
$$

is an equivalence of categories. 
2.8. Let $X$ be a smooth scheme. We call a cdo on $X$ a sheaf of vertex algebras $\mathscr{A}$ over $X$ (in the Zariski topology) such that for every open $U \subset X$ the algebra $\Gamma(U ; \mathscr{A})$ is a cdo over $\Gamma\left(U ; \mathscr{O}_{X}\right)$. Such algebras form a groupoid $\mathscr{C D O} O_{X}$. In a similar manner, one defines a groupoid $\mathscr{A} \lg _{X}$; the (sheafified versions of) functors $\mathscr{A}$ and $U$ determine an equivalence of categories $\mathscr{A l g} \lg _{X} \stackrel{\sim}{\longrightarrow} \mathscr{C D O} O_{X}$.

First we discuss the case of an affine scheme $X=\operatorname{Spec}(A)$. Given a vertex algebroid $\mathscr{A} \in A \lg _{A}$, we may apply the pushout construction 2.4 to all localization morphisms $A \rightarrow A_{f}$ and get a sheaf of vertex algebroids $\mathscr{A}^{\sim}$ over $\operatorname{Spec}(A)$. This gives an equivalence of categories $A \lg _{A} \stackrel{\sim}{\rightarrow} A \lg _{\text {Spec(A) }}$, the inverse equivalence being the functor of global sections.

Returning to the case of an arbitrary $X$, we have a sheaf of categories $\mathscr{D i f f}_{X}^{\text {ch }}$ over $X$ where by definition $\Gamma(U$; $\mathscr{D i f f} X)=\mathscr{A l g} \lg _{U}=\mathscr{C D O O}_{U}$. Consider the truncated de Rham complex $\Omega_{X}^{[2,3\rangle}: \Omega_{X}^{2} \rightarrow \Omega_{X}^{3, \mathrm{cl}}$, with the first sheaf living in degree 0 . By a general homological formalism (cf. [8, 7.3]) we have a canonical characteristic class

$$
c\left(\mathscr{D i f f}{ }_{X}^{\mathrm{ch}}\right) \in H^{2}\left(X ; \Omega_{X}^{[2,3\rangle}\right)
$$

which is zero if and only if $\mathscr{C D O} O_{X}=\varnothing$. The set of isomorphism classes $\pi_{0}(\mathscr{C D O O})$ is a torseur under $H^{1}\left(X ; \Omega_{X}^{[2,3\rangle}\right)$ and the automorphism group of cdo over $X$ is isomorphic to $H^{0}\left(X ; \Omega_{X}^{[2,3\rangle}\right)$.

To compute the class (2.28), note first that we have an obvious map $H^{2}\left(X ; \Omega_{X}^{[2,3\rangle}\right) \rightarrow$ $H^{2}\left(X ; \Omega_{X}^{\geq 2}\right)$ which is easily seen to be an embedding (cf. Subsection 6.1). Here

$$
\Omega_{X}^{\geq i}=\left(\Omega_{X}^{i} \longrightarrow \Omega_{X}^{i+1} \longrightarrow \cdots\right)
$$

with the first sheaf living in degree zero. The collections of spaces $\left\{H^{i}\left(X ; \Omega_{X}^{\geq i}\right)\right\}$ form a cohomology theory which satisfies the Grothendieck axioms for constructing Chern classes. Thus, for each vector bundle $E$ on $X$, its Chern classes $c_{i}(E) \in H^{i}\left(X ; \Omega_{X}^{\geq i}\right)$ are defined; its images in $H^{i}\left(X ; \Omega_{X}^{i}\right)$ are the usual Chern classes "style Hodge."

Now, the following theorem is one of the main results in [8] (see [8, Section 7]).

THEOREM 2.2. The image of $c\left(\mathscr{D i f f} \mathrm{ch}_{X}^{\mathrm{ch}}\right)$ in $H^{2}\left(X ; \Omega_{\bar{X}}^{2}\right)$ is equal to $2 \mathrm{ch}_{2}\left(\Theta_{X}\right):=c_{1}\left(\Theta_{X}\right)^{2}$ $-2 c_{2}\left(\Theta_{X}\right)$, where $\Theta_{X}$ denotes the tangent bundle.

3. Chiral differential operators over an algebraic group. Perfect vertex algebroids over constants.

The discussion below is nothing but the specification of [8, Sections $1,2,3,4]$ to the case $A=\mathbb{C}$.

3.1. Let $\mathfrak{g}$ be a Lie algebra. We need two complexes connected with $\mathfrak{g}$, both concentrated in nonnegative degrees. The first one, $C^{\cdot}(\mathfrak{g})=C^{\cdot}(\mathfrak{g} ; \mathbb{C})$, is the cochain complex of $\mathfrak{g}$ with trivial coefficients. Thus, by definition $C^{i}(\mathfrak{g})=\left(\Lambda^{i} \mathfrak{g}\right)^{*}=$ the space of skew symmetric polylinear maps $f: \mathfrak{g}^{i} \rightarrow \mathbb{C}, i \geq 0$.

The differential $d: C^{i-1}(\mathfrak{g}) \rightarrow C^{i}(\mathfrak{g})$ acts as

$$
d f\left(\tau_{1}, \ldots, \tau_{i}\right)=\sum_{1 \leq p<q \leq i}(-1)^{p+q+1} f\left(\left[\tau_{p}, \tau_{q}\right], \tau_{1}, \ldots, \hat{\tau}_{p}, \ldots, \hat{\tau}_{q}, \ldots, \tau_{i}\right) .
$$

The cohomology spaces $H^{i}\left(C^{\cdot}(\mathfrak{g})\right)$ is denoted by $H^{i}(\mathfrak{g})$. 
The second complex, $\tilde{C} \cdot(\mathfrak{g})$, is the shifted by 1 and augmented cochain complex of $\mathfrak{g}$ with coefficients in the coadjoint representation $\mathfrak{g}^{*}$. By definition, $\tilde{C}^{0}(\mathfrak{g})=\mathbb{C}$ and $\tilde{C}^{i}(\mathfrak{g})=\operatorname{Hom}_{k}\left(\Lambda^{i-1} \mathfrak{g}, \mathfrak{g}^{*}\right)=$ the space of skew symmetric polylinear maps $h: \mathfrak{g}^{i-1} \rightarrow \mathfrak{g}^{*}$ for $i \geq 1$.

The differential $d: \tilde{C}^{0}(\mathfrak{g}) \rightarrow \tilde{C}^{1}(\mathfrak{g})$ is zero, and $d: \tilde{C}^{i}(\mathfrak{g}) \rightarrow \tilde{C}^{i+1}(\mathfrak{g})$ acts as

$$
\begin{aligned}
d h\left(\tau_{1}, \ldots, \tau_{i}\right)= & \sum_{p=1}^{i}(-1)^{p} \tau_{p}\left(h\left(\tau_{1}, \ldots, \hat{\tau}_{p}, \ldots, \tau_{i}\right)\right) \\
& +\sum_{1 \leq p<q \leq i}(-1)^{p+q} h\left(\left[\tau_{p}, \boldsymbol{\tau}_{q}\right], \boldsymbol{\tau}_{1}, \ldots, \hat{\tau}_{p}, \ldots, \hat{\tau}_{q}, \ldots, \boldsymbol{\tau}_{i}\right)
\end{aligned}
$$

for $i \geq 1$. Define embeddings $C^{i}(\mathfrak{g}) \hookrightarrow \tilde{C}^{i}(\mathfrak{g})$ by assigning to $f \in C^{i}(\mathfrak{g})$ an element $\tilde{f} \in \tilde{C}^{i}(\mathfrak{g})$ given by

$$
\left\langle\tau_{1}, \tilde{f}\left(\tau_{2}, \ldots, \tau_{i}\right)\right\rangle=f\left(\tau_{1}, \ldots, \tau_{i}\right) .
$$

We identify $C^{i}(\mathfrak{g})$ with its image in $\tilde{C}^{i}(\mathfrak{g})$.

One checks that the embeddings (3.3) are compatible with the differentials, so that one has an embedding of complexes $C^{\cdot}(\mathfrak{g}) \hookrightarrow \tilde{C}^{\cdot}(\mathfrak{g})$.

3.2. We consider the groupoid $A \lg _{\mathfrak{g}}$ of vertex algebroids of the form $\mathscr{A}=$ $\left(\mathbb{C}, \mathfrak{g}, \mathfrak{g}^{*}, \partial, \gamma,\langle\cdot, \cdot\rangle, c\right)$, where $\mathscr{T}=(\mathbb{C}, T, \Omega, \partial)=\left(\mathbb{C}, \mathfrak{g}, \mathfrak{g}^{*}, 0\right)$ is a perfect extended Lie algebroid over $\mathbb{C}$, see Subsection 2.2 , with $T=\mathfrak{g}$. Note that the last object is uniquely defined by the Lie algebra $\mathfrak{g}=T$; we must have $\Omega=\mathfrak{g}^{*}$, the "Lie derivative" action of $T$ on $\Omega$ must be the coadjoint one, and a $\mathbb{C}$-linear derivation $\partial: \mathbb{C} \rightarrow \Omega$ must be zero.

Turning to the axioms of a vertex algebroid, Subsection 2.3, we see that for $\mathscr{A}$ as above, $\langle\cdot, \cdot\rangle: \mathfrak{g} \times \mathfrak{g} \rightarrow \mathbb{C}$ is a symmetric bilinear map (which may be regarded as an element of $\left.\tilde{C}^{2}(\mathfrak{g})\right), c \in \tilde{C}^{3}(\mathfrak{g})$, equation (2.7) implies that $\gamma=0$, (2.8) and (2.9) hold true automatically, (2.10) takes the form

$$
\left\langle\left[\tau_{1}, \tau_{2}\right], \tau_{3}\right\rangle+\left\langle\tau_{2},\left[\tau_{1}, \tau_{3}\right]\right\rangle=\left\langle\tau_{2}, c\left(\tau_{1}, \tau_{3}\right)\right\rangle+\left\langle\tau_{3}, c\left(\tau_{1}, \tau_{2}\right)\right\rangle,
$$

and (2.11) takes the form

$$
d c=0,
$$

where $d$ is the differential in $\tilde{C}^{\cdot}(\mathfrak{g})$ given by (3.2).

So, an object of $\mathscr{A} \lg _{\mathfrak{g}}$ has a form

$$
\mathscr{A}_{\mathfrak{g} ;\langle\cdot, \cdot\rangle, c}=\left(\mathbb{C}, \mathfrak{g}, \mathfrak{g}^{*}, 0,0,\langle\cdot, \cdot\rangle, c\right),
$$

where $\langle\cdot, \cdot\rangle \in \tilde{C}^{2}(\mathfrak{g})^{\mathbb{Z} / 2 \mathbb{Z}}, c \in \tilde{C}^{3}(\mathfrak{g})$ satisfy (3.4) and (3.5).

The vertex envelope

$$
\mathscr{V}_{\mathfrak{g} ;\langle\cdot, \cdot\rangle, c}=U \mathscr{A}_{\mathfrak{g} ;\langle\cdot, \cdot\rangle, c}
$$

(see [8, Section 9]) is generated by the fields $\tau(z)(\tau \in \mathfrak{g})$ and $\omega(z)\left(\omega \in \mathfrak{g}^{*}\right)$ of conformal weight 1 , subject to operator product expression (OPE)

$$
\begin{gathered}
\tau(z) \tau^{\prime}(w) \sim \frac{\left\langle\tau, \tau^{\prime}\right\rangle}{(z-w)^{2}}+\frac{\left[\tau, \tau^{\prime}\right](w)-c\left(\tau, \tau^{\prime}\right)(w)}{z-w}, \\
\tau(z) \omega(w) \sim \frac{\tau(\omega)(w)}{z-w} ; \quad \omega(z) \omega^{\prime}(w) \sim 0
\end{gathered}
$$

(cf. [8, (9.9.1)-(9.9.3)]). 
A morphism

$$
f: \mathscr{A}_{\mathfrak{g},\langle\cdot, \cdot\rangle, c} \longrightarrow \mathscr{A}_{\mathfrak{g},\langle\cdot, \cdot\rangle^{\prime}, c^{\prime}}
$$

is by definition an element $h \in \tilde{C}^{2}(\mathfrak{g})$ such that

$$
\left\langle\tau_{1}, h\left(\tau_{2}\right)\right\rangle+\left\langle\tau_{2}, h\left(\tau_{1}\right)\right\rangle=\left\langle\tau_{1}, \tau_{2}\right\rangle-\left\langle\tau_{1}, \tau_{2}\right\rangle^{\prime}, \quad d h=c-c^{\prime},
$$

see Subsection 2.5. The composition of morphisms is induced by the addition in $\tilde{C}^{2}(\mathfrak{g})$.

3.3. As a corollary, we have a canonical bijection

$$
\pi_{0}\left(\mathscr{A} \lg _{\mathfrak{g}}\right)=H^{3}(\mathfrak{g}) .
$$

More precisely, for a 3-cocycle $c \in C^{3, \mathrm{cl}}(\mathfrak{g})$ we have a vertex algebroid

$$
\mathscr{A}_{\mathfrak{g} ; c}:=\mathscr{A}_{\mathfrak{g} ; 0, c},
$$

and the correspondence $c \mapsto \mathscr{A}_{\mathfrak{g} ; c}$ induces the bijection (3.12).

The enveloping algebra $\mathscr{V}_{\mathfrak{g} ; c}:=U A_{\mathfrak{g} ; c}$ is generated by the same fields as in Subsection 3.2, subject to OPE

$$
\tau(z) \tau^{\prime}(w) \sim \frac{\left[\tau, \tau^{\prime}\right](w)-c\left(\tau, \tau^{\prime}\right)(w)}{z-w},
$$

and (3.9).

We define another interesting class of objects of $A_{\lg }$. Namely, each symmetric adinvariant bilinear form $(\cdot, \cdot) \in\left(S^{2} \mathfrak{g}^{*}\right)^{\mathfrak{g}}$ gives rise to an object

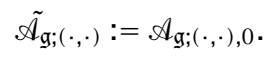

The enveloping algebra $\tilde{\mathscr{V}}_{\mathrm{g} ;(\cdot, \cdot)}:=U \tilde{A}_{\mathfrak{g} ;(\cdot, \cdot)}$ is generated by the same fields as in Subsection 3.2, subject to OPE

$$
\tau(z) \tau^{\prime}(w) \sim \frac{\left(\tau, \tau^{\prime}\right)}{(z-w)^{2}}+\frac{\left[\tau, \tau^{\prime}\right](w)}{z-w}
$$

and (3.9).

It is easy to see that, we have an isomorphism

$$
f_{(\cdot, \cdot)}: \tilde{A}_{\mathfrak{g} ;(\cdot, \cdot)} \stackrel{\sim}{\longrightarrow} \mathscr{A}_{\mathfrak{g} ; c_{(\cdot, \cdot)}}
$$

given by a map $h_{(\cdot, \cdot)}: \mathfrak{g} \rightarrow \mathfrak{g}^{*}$, where

$$
\left\langle\tau_{1}, h_{(\cdot, \cdot)}\left(\tau_{2}\right)\right\rangle=\frac{1}{2}\left(\tau_{1}, \tau_{2}\right),
$$

and the cocycle $c_{(\cdot, \cdot)}$ is defined by

$$
c_{(\cdot, \cdot)}\left(\tau_{1}, \tau_{2}, \tau_{3}\right)=\left(\left[\tau_{1}, \tau_{2}\right], \tau_{3}\right)
$$

(cf. [8, Theorem 4.5]). 
3.4. Given $(\cdot, \cdot) \in\left(S^{2} \mathfrak{g}^{*}\right)^{\mathfrak{g}}$, consider a vertex algebroid

$$
\mathscr{A}_{\mathfrak{g} ;(\cdot, \cdot)}:=\left(\mathbb{C}, \mathfrak{g}, \mathfrak{g}^{*}, 0,0,(\cdot, \cdot), 0\right) .
$$

Its vertex envelope $\mathscr{V}_{\mathfrak{g} ;(\cdot, \cdot)}:=U \mathscr{A}_{\mathfrak{g} ;(\cdot, \cdot)}$ is generated by fields $\tau(z)(\tau \in \mathfrak{g})$ of conformal weight 1 , subject to OPE (3.16).

The correspondence $\boldsymbol{\tau} \cdot T^{n} \mapsto \boldsymbol{T}_{(n)}$ defines on $\mathscr{V}_{\mathfrak{g} ;(\cdot, \cdot)}$ a structure of the vacuum module over the Kac-Moody algebra $\hat{\mathfrak{g}}=\mathfrak{g}\left[T, T^{-1}\right] \oplus \mathbb{C} \cdot \mathbf{1}$ at level $(\cdot, \cdot)$.

We have an obvious embedding of vertex algebroids $A_{\mathfrak{g} ;(\cdot, \cdot)} \hookrightarrow \mathscr{A}_{\mathfrak{g} ; 0}$ which induces an embedding of vertex algebras

$$
\mathscr{V}_{\mathfrak{g} ;(\cdot, \cdot)} \longrightarrow \tilde{V}_{\mathfrak{g} ;(\cdot, \cdot)}
$$

REMARK 3.1. If the Lie algebra $\mathfrak{g}$ is semi-simple then the correspondence $(\cdot, \cdot) \mapsto$ $c_{(\cdot, \cdot)}$ induces a bijection

$$
\left(S^{2} \mathfrak{g}^{*}\right)^{\mathfrak{g}} \stackrel{\sim}{\longrightarrow} H^{3}(\mathfrak{g}) .
$$

Therefore, in this case the algebroids $\tilde{\mathscr{A}_{\mathfrak{g} ;(\cdot, \cdot)}}$ form a complete set of representatives of isomorphism classes in $A \lg _{\mathfrak{g}}$. In other words,

(1) if $\mathfrak{g}$ is semi-simple, then the correspondence $(\cdot, \cdot) \mapsto \tilde{A_{\mathfrak{g} ;(\cdot, \cdot)}}$ induces a bijection

$$
\left(S^{2} \mathfrak{g}^{*}\right)^{\mathfrak{g}} \stackrel{\sim}{\longrightarrow} \pi_{0}\left(A \lg _{\mathfrak{g}}\right)
$$

passing to a group.

3.5. Let $G=\operatorname{Spec}(A)$ be an affine algebraic group, and let $\mathfrak{g}$ be the corresponding Lie algebra. The tangent bundle $\Theta_{G}$ is trivial, so the obstruction $c\left(\mathscr{D i f f}_{G}^{\mathrm{ch}}\right)$ to the existence of a cdo over $G, \mathscr{D}_{G}^{\mathrm{ch}} \in \Gamma\left(G\right.$; $\left.\mathfrak{D i f f}_{G}^{\mathrm{ch}}\right)$ (cf. [8, Corollary 7.11]) vanishes.

From Section 4 , the set of isomorphism classes of cdo's over $G, \pi_{0}\left(\Gamma\left(G ; \mathfrak{D i f f}_{G}^{\mathrm{ch}}\right)\right)$ is a nonempty torseur under the "Chern-Simons group" $H_{\mathrm{DR}}^{3}(G)=H^{3}(G ; \mathbb{C})$.

In fact the groupoid $\Gamma\left(G ; \mathscr{D i f f}{ }_{G}^{\mathrm{ch}}\right)$ has a distinguished object $\mathscr{D}_{G ; 0}^{\mathrm{ch}}$, so that we have a canonical bijection

$$
\pi_{0}\left(\Gamma\left(G ; \text { Diff }{ }_{G}^{\mathrm{ch}}\right)\right) \stackrel{\sim}{\longrightarrow} H_{\mathrm{DR}}^{3}(G) .
$$

This is a consequence of the following general construction.

3.6. Let $\mathscr{A}_{\mathfrak{g} ;\langle\cdot, \cdot\rangle, \mathrm{c}}$ be an arbitrary object of $\mathscr{A l g}_{\mathfrak{g}}$. We apply to it the pushout construction of Subsection 2.4 with respect to the structure morphism $\mathbb{C} \rightarrow A$. Here the morphism $\mathfrak{g} \rightarrow T:=\operatorname{Der}_{\mathbb{C}}(A)$ is defined as the embedding of left invariant vector fields, and the map $\gamma: A \times \mathfrak{g} \rightarrow \Omega:=\Omega^{1}(A)$ is set to be zero. This way we get a vertex $A$-algebroid $A_{G ;\langle\cdot, \cdot, c}$. Its enveloping algebra

$$
\mathscr{D}_{G ;\langle\cdot, \cdot\rangle, c}^{\mathrm{ch}}=U \mathscr{A}_{G ;\langle\cdot, \cdot\rangle, c}
$$

obviously belongs to $\Gamma\left(G\right.$; $\left.\mathscr{D i f f}{ }_{G}^{\mathrm{ch}}\right)$.

We have a canonical embedding

$$
\mathscr{V}_{\mathfrak{g},\langle\cdot, \cdot\rangle, c} \longleftrightarrow \mathscr{D}_{G ;\langle\cdot, \cdot\rangle, c}^{\mathrm{ch}} .
$$


We use the notation $\mathscr{A}_{G ;(\cdot, \cdot)}:=\mathscr{A}_{G ;(\cdot, \cdot), 0}, \mathscr{A}_{G ; c}:=\mathscr{A}_{G ; 0, c}, \mathscr{A}_{G ; 0}:=\mathscr{A}_{G ; 0,0}$, and $\mathscr{D}_{G ;(\cdot,)}^{\mathrm{ch}}$, etc. for the corresponding enveloping algebras.

If $\mathscr{A}_{G ; 0}=\left(A, T, \Omega, d_{\mathrm{DR}}, \gamma_{0},\langle\cdot, \cdot\rangle_{0}, \mathcal{C}_{0}\right)$ and $\omega \in \Omega^{3, \mathrm{cl}}(A)$ is a closed 3-form, then we can form a vertex algebroid

$$
\mathscr{A}_{G ; \omega}:=\mathscr{A}_{G ; 0}+\omega=\left(A, T, \Omega, d_{\mathrm{DR}}, \gamma_{0},\langle\cdot, \cdot\rangle_{0}, c_{0}+\omega\right) .
$$

The correspondence $\omega \mapsto \mathscr{A}_{G ; \omega}$ induces the bijection (3.24).

If $c \in C^{3, c l}(\mathfrak{g})$ is a 3-cocycle with trivial coefficients, then by definition

$$
\mathscr{A}_{G ; c}=\mathscr{A}_{G ; \omega_{c}},
$$

where $\omega_{c} \in \Omega^{3, \mathrm{cl}}(A)$ is the left invariant 3-form on $G$ corresponding to $c$.

COROLlary 3.2. Assume that $G$ is reductive. Then the correspondence $c \mapsto A_{G ; c}$ induces a bijection

$$
H^{3}(\mathfrak{g}) \stackrel{\sim}{\longrightarrow} \pi_{0}\left(\Gamma\left(G ; \text {Diff }_{G}^{\mathrm{ch}}\right)\right) .
$$

Indeed, one knows that for a reductive group the correspondence $c \mapsto \omega_{c}$ gives rise to an isomorphism $H^{3}(\mathfrak{g}) \stackrel{\sim}{\longrightarrow} H_{\mathrm{DR}}^{3}(G)$.

Corollary 3.3. Assume that $G$ is semi-simple. Then the correspondence $(\cdot, \cdot) \mapsto$ $A_{G ;(\cdot, \cdot)}$ induces a bijection

$$
\left(S^{2} \mathfrak{g}^{*}\right)^{\mathfrak{g}} \stackrel{\sim}{\longrightarrow} \pi_{0}\left(\Gamma\left(G ; \text {Diff }_{G}^{\mathrm{ch}}\right)\right) .
$$

This follows from Corollary 3.2 and Remark 3.1.

3.7. Note that for an arbitrary $G$ and $(\cdot, \cdot) \in\left(S^{2} \mathfrak{g}^{*}\right)^{\mathfrak{g}}$ one has a canonical embedding

$$
i_{(\cdot, \cdot)}: \mathscr{V}_{\mathfrak{g} ;(\cdot, \cdot)} \longrightarrow \mathscr{D}_{G ;(\cdot, \cdot)}^{\mathrm{ch}} .
$$

It is the composition of (3.21) and (3.26).

\section{Dual embedding}

4.1. Let $G=\operatorname{Spec}(A)$ be a smooth affine connected algebraic group with the Lie algebra $\mathfrak{g}$. Pick a symmetric ad-invariant bilinear form ("level”) $(\cdot, \cdot) \in\left(S^{2} \mathfrak{g}^{*}\right)^{\mathfrak{g}}$.

Let $(\cdot, \cdot)_{(K)}$ denote the Killing form on $\mathfrak{g}$,

$$
(x, y)_{(K)}=\operatorname{tr}_{\mathfrak{g}}\left(\operatorname{ad}_{x} \cdot \operatorname{ad}_{y}\right) .
$$

We pick a base $\left\{\boldsymbol{\tau}_{i}\right\}$ of $\mathfrak{g}$. In terms of structure constants

$$
\left[\tau_{i}, \tau_{j}\right]=c_{p}^{i j} \tau_{p}
$$

the form (4.1) is given by

$$
\left(\boldsymbol{T}_{i}, \boldsymbol{T}_{j}\right)_{(K)}=c_{q}^{i p} c_{p}^{j q} .
$$

We define the dual level $(\cdot, \cdot)^{o} \in\left(S^{2} \mathfrak{g}^{*}\right)^{\mathfrak{g}}$ by

$$
(\cdot, \cdot)^{o}=-(\cdot, \cdot)_{(K)}-(\cdot, \cdot) .
$$


Define the critical level $(\cdot, \cdot)_{\text {crit }}$ by $(\cdot, \cdot)_{\text {crit }}=(\cdot, \cdot)_{\text {crit }}^{o}$, that is,

$$
(\cdot, \cdot)_{\text {crit }}=-\frac{1}{2}(\cdot, \cdot)_{(K)} \text {. }
$$

If we want to stress the dependence on $\mathfrak{g}$, we write $(\cdot, \cdot)_{\mathfrak{g} ;(K)},(\cdot, \cdot)_{\mathfrak{g} ; \text { crit }}$.

4.2. We have two commuting left actions of $G$ on itself: the left multiplication, $(g, x) \mapsto g x$ and the right one, $(g, x) \mapsto x g^{-1}$.

Let $T=\operatorname{Der}_{k}(A)$ denote the Lie $A$-algebroid of vector fields over $G$. The above two actions induce two embeddings of Lie algebras

$$
i_{L}: \mathfrak{g} \longleftrightarrow T, \quad i_{R}: \mathfrak{g} \longleftrightarrow T,
$$

such that

$$
\left[i_{L}(x), i_{R}(y)\right]=0 \quad \forall x, y \in \mathfrak{g} .
$$

Below we identify $\mathfrak{g}$ with its image under $i_{L}$, that is, write simply $x$ instead of $i_{L}(x)$. We also use the notation $x^{R}:=i_{R}(x)(x \in \mathfrak{g})$.

Embedding $i_{L}$ induces an isomorphism of left $A$-modules

$$
A \otimes_{k} \mathfrak{g} \stackrel{\sim}{\longrightarrow} T .
$$

Thus, $\left\{\boldsymbol{\tau}_{i}\right\}$ form an $A$-base of $T$. In particular,

$$
\tau_{i}^{R}=a^{i j} \tau_{j}
$$

for some invertible matrix $\left(a^{i j}\right)$ over $A$.

The commutation relations

$$
\left[\tau_{i}, \tau_{j}^{R}\right]=0
$$

are equivalent to the identities

$$
\tau_{i}\left(a^{j s}\right)+c_{s}^{i p} a^{j p}=0,
$$

true for all $i, j, s$.

We write down the relations

$$
\left[\tau_{i}^{R}, \tau_{j}^{R}\right]=\left[\tau_{i}, \tau_{j}\right]^{R}
$$

in coordinates. We have

$$
\left[\tau_{i}^{R}, \tau_{j}^{R}\right]=\left[\tau_{i}^{R}, a^{j s} \tau_{s}\right]=\tau_{i}^{R}\left(a^{j s}\right) \tau_{s}=a^{i p} \tau_{p}\left(a^{j s}\right) \tau_{s}
$$

due to (4.10). Plugging this into (4.12), we get

$$
a^{i p} \tau_{p}\left(a^{j s}\right)=c_{q}^{i j} a^{q s} \quad \forall i, j, s .
$$


4.3. We set $\Omega:=\Omega_{A / k}^{1}=\operatorname{Hom}_{A}(T, A)$, and denote by $\langle\cdot, \cdot\rangle: T \times \Omega \rightarrow A$ the canonical $A$-bilinear pairing. Let $\left\{\omega_{i}\right\}$ be the $A$-base of $\Omega$ dual to $\left\{\tau_{i}\right\}$. The Lie algebra $T$ acts on $\Omega$ by the Lie derivative.

We have $\tau_{i}\left(\omega_{j}\right)=\alpha^{i j s} \omega_{s}$, where

$$
\alpha^{i j s}=\left\langle\tau_{s}, \tau_{i}\left(\omega_{j}\right)\right\rangle=\tau_{i}\left(\left\langle\tau_{s}, \omega_{j}\right\rangle\right)-\left\langle\left[\tau_{i}, \tau_{s}\right], \omega_{j}\right\rangle=-c_{j}^{i s}=c_{j}^{s i} .
$$

Thus,

$$
\tau_{i}\left(\omega_{j}\right)=c_{j}^{s i} \omega_{s}
$$

Similarly,

$$
\tau_{i}^{R}\left(\omega_{j}\right)=0
$$

4.4. Recall that we have an embedding of vertex algebras

$$
i_{(\cdot, \cdot)}: \mathscr{V}_{\mathfrak{g} ;(\cdot, \cdot)} \longleftrightarrow \mathscr{D}_{G ;(\cdot, \cdot)}^{\mathrm{ch}},
$$

see Subsection 3.7. More precisely, it is induced by an embedding of conformal weight 1 components

$$
j_{L}: \mathfrak{g}=\mathscr{V}_{\mathfrak{g},(\cdot, \cdot) 1} \longrightarrow \mathscr{D}_{G ;(\cdot, \cdot) 1}^{\mathrm{ch}}=T \oplus \Omega
$$

defined by a composition

$$
\mathfrak{g} \longleftrightarrow T \longleftrightarrow T \oplus \Omega
$$

where the first arrow is $i_{L}$ and the second one sends $x$ to $(x, 0)$.

The fact that $j_{L}$ induces a map of vertex algebras (4.18) simply means that, we have the identities in $\mathscr{D}_{G ;(\cdot,)}^{\mathrm{ch}}$

$$
j_{L}(\tau)_{(1)} j_{L}\left(\tau^{\prime}\right)=\left(\tau, \tau^{\prime}\right) ; \quad j_{L}(\tau)_{(0)} j_{L}\left(\tau^{\prime}\right)=j_{L}\left(\left[\tau, \tau^{\prime}\right]\right), \quad \forall \tau, \tau^{\prime} \in \mathfrak{g} .
$$

ThEOREM 4.1 (B. Feigin-E. Frenkel, D. Gaitsgory). (i) There exists a unique embedding

$$
j_{R}: \mathfrak{g} C \mathscr{D}_{G ;(\cdot, \cdot) 1}^{\mathrm{ch}}
$$

such that

(a) the composition of (4.22) with the canonical projection $\mathscr{D}_{G ;(\cdot, \cdot) 1}^{\mathrm{ch}} \rightarrow T$ is equal to $i_{R}$;

(b) for all $\tau, \tau^{\prime} \in \mathfrak{g}$ and $n \geq 0$

$$
j_{L}(\tau)_{(n)} j_{R}\left(\tau^{\prime}\right)=0
$$

(ii) We have

$$
\begin{gathered}
j_{R}(\tau)_{(1)} j_{R}\left(\tau^{\prime}\right)=\left(\tau, \tau^{\prime}\right)^{o}, \\
j_{R}(\tau)_{(0)} j_{R}\left(\tau^{\prime}\right)=j_{R}\left(\left[\tau, \tau^{\prime}\right]\right),
\end{gathered}
$$

for each $\tau, \tau^{\prime} \in \mathfrak{g}$. 
COROLLARY 4.2 (B. Feigin-E. Frenkel, D. Gaitsgory). The map (4.22) induces an embedding of chiral algebras

$$
j_{R}: \mathscr{V}_{\mathfrak{g} ;(\cdot, \cdot)^{o}} \longleftrightarrow \mathscr{D}_{G ;(\cdot, \cdot)}^{\mathrm{ch}} .
$$

The images of $j_{L}$ and $j_{R}$ commute in the following sense:

$$
j_{L}(x)_{(n)} j_{R}(y)=0
$$

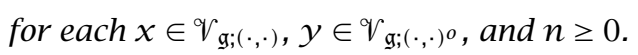

Proof of TheOrem 4.1. (i) As usual, we denote $j_{L}(\tau)$ simply by $\tau$. We are looking for $j_{R}(\tau)$ in the form

$$
j_{R}\left(\tau_{i}\right)=\tau_{i}^{R}+b^{i q} \omega_{q}
$$

for some $b^{i q} \in A$. We have, by $[8,1.4(\mathrm{~A} 2)]$,

$$
\tau_{i(1)}^{R} \tau_{j}=\left\langle\tau_{i}^{R}, \tau_{j}\right\rangle=\left\langle a^{i p} \tau_{p}, \tau_{j}\right\rangle=a^{i p}\left(\tau_{p}, \tau_{j}\right)-\tau_{p} \tau_{j}\left(a^{i p}\right) .
$$

Using (4.4) and (4.11),

$$
-\tau_{p} \tau_{j}\left(a^{i p}\right)=\tau_{p}\left(c_{p}^{j s} a^{i s}\right)=-c_{p}^{p u} c_{p}^{j s} a^{i u}=c_{s}^{u p} c_{p}^{j s} a^{i u}=\left(\tau_{u}, \tau_{j}\right)_{(K)} a^{i u},
$$

so

$$
\tau_{i(1)}^{R} \tau_{j}=-\left(\tau_{p}, \tau_{j}\right)^{o} a^{i p} .
$$

On the other hand,

$$
\left(b^{i q} \omega_{q}\right)_{(1)} \tau_{j}=\left\langle b^{i q} \omega_{q}, \tau_{j}\right\rangle=b^{i j} .
$$

Therefore, the condition

$$
j_{R}\left(\tau_{i}\right)_{(1)} \tau_{j}=0
$$

defines the matrix $\left(b^{i q}\right)$ uniquely, we must have

$$
j_{R}\left(\tau_{i}\right)=\tau_{i}^{R}+\left(\tau_{p}, \tau_{q}\right)^{o} a^{i p} \omega_{q} .
$$

We prove that

$$
\tau_{i(0)} j_{R}\left(\tau_{j}\right)=0
$$

We have

$$
\tau_{i(0)} j_{R}\left(\tau_{j}\right)=\tau_{i(0)}\left\{\tau_{j}^{R}+\left(\tau_{s}, \tau_{u}\right)^{o} a^{j s} \omega_{u}\right\}
$$

On one hand,

$$
\boldsymbol{T}_{i(0)} \boldsymbol{\tau}_{j}^{R}=\boldsymbol{T}_{i(0)}\left(a^{j q} \boldsymbol{T}_{q}\right)=\boldsymbol{\tau}_{i(0)}\left(a_{(-1)}^{j q} \boldsymbol{T}_{q}\right)=\boldsymbol{\tau}_{i}\left(a^{j q}\right)_{(-1)} \boldsymbol{T}_{q}+a_{(-1)}^{j q}\left[\boldsymbol{\tau}_{i}, \boldsymbol{\tau}_{q}\right]=0,
$$

using (4.11). On the other hand,

$$
\begin{aligned}
\boldsymbol{T}_{i(0)} & \left\{\left(\boldsymbol{\tau}_{s}, \boldsymbol{\tau}_{u}\right)^{o} a^{j s} \omega_{u}\right\} \\
& =\left(\boldsymbol{\tau}_{s}, \boldsymbol{\tau}_{u}\right)^{o} \boldsymbol{\tau}_{i}\left(a^{j s} \omega_{u}\right) \\
& =\left(\boldsymbol{\tau}_{s}, \boldsymbol{\tau}_{u}\right)^{o}\left\{\boldsymbol{\tau}_{i}\left(a^{j s}\right) \omega_{u}+a^{j s} \boldsymbol{\tau}_{i}\left(\omega_{u}\right)\right\} \\
& =-\left(\boldsymbol{\tau}_{s}, \boldsymbol{\tau}_{p}\right)^{o} c_{s}^{i q} a^{j q} \omega_{p}-\left(\boldsymbol{\tau}_{s}, \boldsymbol{\tau}_{u}\right)^{o} c_{u}^{i p} a^{j s} \omega_{p} \quad \text { using (4.11) and (4.16) } \\
& =-\left(\left[\boldsymbol{\tau}_{i}, \boldsymbol{\tau}_{q}\right], \boldsymbol{\tau}_{p}\right)^{o} a^{j q} \omega_{p}-\left(\boldsymbol{\tau}_{s},\left[\boldsymbol{\tau}_{i}, \boldsymbol{\tau}_{p}\right]\right)^{o} a^{j s} \omega_{p}=0
\end{aligned}
$$

due to the invariance of the form $(\cdot, \cdot)^{o}$. This proves $(4.35)$. 
Evidently $\boldsymbol{\tau}_{i(n)} j_{R}\left(\boldsymbol{\tau}_{j}\right)=0$ for $n \geq 2$. This proves part (i) of the theorem.

We compute $j_{R}\left(\tau_{i}\right)_{(1)} j_{R}\left(\tau_{j}\right)$. We have

$$
j_{R}\left(\tau_{i}\right)_{(1)} j_{R}\left(\tau_{j}\right)=\left\{\tau_{i}^{R}+\left(\tau_{p}, \tau_{q}\right)^{o} a^{i p} \omega_{q}\right\}_{(1)}\left\{\tau_{j}^{R}+\left(\tau_{s}, \tau_{u}\right)^{o} a^{j s} \omega_{u}\right\} .
$$

This is a sum of four terms,

$$
\begin{aligned}
\mathrm{I}:= & \tau_{i(1)}^{R} \boldsymbol{\tau}_{j}^{R}=\left\langle a^{i p} \tau_{p}, a^{j s} \tau_{s}\right\rangle \\
= & a^{i p} a^{j s}\left(\tau_{p}, \boldsymbol{\tau}_{s}\right)-a^{i p} \tau_{s} \tau_{p}\left(a^{j s}\right)-a^{j s} \tau_{p} \tau_{s}\left(a^{i p}\right) \\
& -\tau_{p}\left(a^{j s}\right) \tau_{s}\left(a^{i p}\right) \quad \text { using }\left[8,(1.8 .3)_{\langle,\rangle}\right] .
\end{aligned}
$$

Using (4.4) and (4.11) we see that

$$
-a^{i p} \tau_{s} \tau_{p}\left(a^{j s}\right)=-a^{j s} \tau_{p} \tau_{s}\left(a^{i p}\right)=\tau_{p}\left(a^{j s}\right) \tau_{s}\left(a^{i p}\right)=\left(\tau_{p}, \tau_{s}\right)_{(K)} a^{i p} a^{j s},
$$

whence

$$
\mathrm{I}=-\left(\tau_{p}, \tau_{s}\right)^{o} a^{i p} a^{j s} .
$$

Next,

$$
\mathrm{II}:=\left\{\left(\tau_{p}, \tau_{q}\right)^{o} a^{i p} \omega_{q}\right\}_{(1)} \tau_{j}^{R}=\left(\tau_{p}, \tau_{q}\right)^{o}\left\langle a^{i p} \omega_{q}, a^{j s} \tau_{s}\right\rangle=\left(\tau_{p}, \tau_{q}\right)^{o} a^{i p} a^{j q} .
$$

Similarly,

$$
\mathrm{III}:=\tau_{i(1)}^{R}\left\{\left(\tau_{s}, \tau_{u}\right)^{o} a^{j s} \omega_{u}\right\}=\mathrm{II},
$$

and evidently

$$
\mathrm{IV}:=\left\{\left(\tau_{p}, \tau_{q}\right)^{o} a^{i p} \omega_{q}\right\}_{(1)}\left\{\left(\tau_{s}, \tau_{u}\right)^{o} a^{j s} \omega_{u}\right\}=0 .
$$

Adding up, we get

$$
j_{R}\left(\tau_{i}\right)_{(1)} j_{R}\left(\tau_{j}\right)=\left(\tau_{p}, \tau_{q}\right)^{o} a^{i p} a^{j q} .
$$

We differentiate this statement. We have

$$
\begin{aligned}
\tau_{s}\left\{\left(\tau_{p}, \tau_{q}\right)^{o} a^{i p} a^{j q}\right\} & =\left(\tau_{p}, \tau_{q}\right)^{o}\left\{\tau_{s}\left(a^{i p}\right) a^{j q}+a^{i p} \tau_{s}\left(a^{j q}\right)\right\} \\
& =\left(\tau_{p}, \tau_{q}\right)^{o}\left\{-c_{p}^{s u} a^{i u} a^{j q}-c^{s v q} a^{i p} a^{j v}\right\} \\
& =-\left(\left[\tau_{s}, \tau_{u}\right], \tau_{q}\right)^{o} a^{i u} a^{j q}-\left(\tau_{p},\left[\tau_{s}, \tau_{v}\right]\right)^{o} a^{i p} a^{j v} \\
& =0 .
\end{aligned}
$$

Therefore, equation (4.46) is a constant. It may be computed by noticing that the matrix $\left(a^{i j}\right)$, considered as a function on the group $G$, is equal to the identity at the identity of the group. Hence (4.46) is equal to $\left(\boldsymbol{T}_{i}, \boldsymbol{T}_{j}\right)^{o}$, which proves (4.24).

We compute

$$
j_{R}\left(\tau_{i}\right)_{(0)} j_{R}\left(\tau_{j}\right) \in \mathscr{D}_{G ;(\cdot, \cdot) 1}^{\mathrm{ch}}=T \oplus \Omega .
$$

We have

$$
j_{R}\left(\tau_{i}\right)_{(0)} j_{R}\left(\tau_{j}\right)=j_{R}\left(\tau_{i}\right)_{(0)}\left\{a^{j q} \tau_{q}+\left(\tau_{s}, \tau_{u}\right)^{o} a^{j s} \omega_{u}\right\}
$$


We compute the first summand. Using (4.35), we have

$$
j_{R}\left(\boldsymbol{\tau}_{i}\right)_{(0)}\left(a^{j q} \boldsymbol{\tau}_{q}\right)=\left(j_{R}\left(\boldsymbol{\tau}_{i}\right)_{(0)} a^{j q}\right) \tau_{q}=a^{i p} \tau_{p}\left(a^{j q}\right) \tau_{q}=\left[\tau_{i}^{R}, \tau_{j}^{R}\right]=\left[\boldsymbol{\tau}_{i}, \boldsymbol{\tau}_{j}\right]^{R}
$$

by (4.14) and (4.17).

On the other hand,

$$
\begin{aligned}
j_{R}\left(\boldsymbol{T}_{i}\right)_{(0)} & \left\{\left(\tau_{s}, \tau_{u}\right)^{o} a^{j s} \omega_{u}\right\} \\
= & \tau_{i(0)}^{R}\left\{\left(\tau_{s}, \tau_{u}\right)^{o} a^{j s} \omega_{u}\right\}=\left(\tau_{s}, \tau_{u}\right)^{o} \tau_{i}^{R}\left(a^{j s} \omega_{u}\right) \\
= & \left(\tau_{s}, \tau_{u}\right)^{o} a^{i p} \tau_{p}\left(a^{j s}\right) \omega_{u}=\left(\tau_{s}, \tau_{u}\right)^{o} c_{q}^{i j} a^{q s} \omega_{u} \quad \text { by (4.17) and (4.14). }
\end{aligned}
$$

Adding up (4.50) and (4.51) we see that

$$
j_{R}\left(\tau_{i}\right)_{(0)} j_{R}\left(\tau_{j}\right)=j_{R}\left(\left[\tau_{i}, \tau_{j}\right]\right)
$$

which proves (4.25) and part (ii) of the theorem.

Proof OF Corollary 4.2. The first claim is a reformulation of (4.24) and (4.25).

The second claim is a trivial consequence of (4.23) and two Borcherds' formulas

$$
x_{(n)} y_{(-1)} z=y_{(-1)} x_{(n)} z+\sum_{j=0}^{n}\left(\begin{array}{c}
n \\
j
\end{array}\right)\left(x_{(j)} y\right)_{(n-1-j)} z \quad(n \geq 0),
$$

(cf. [8, (0.5.12)]), and

$$
\left(x_{(-1)} y\right)_{(n)} z=\sum_{j \geq 0}\left\{x_{(-1-j)} y_{(n+j)} z+y_{(-n-1-j)} x_{(j)} z\right\}
$$

(cf. $[8,(0.5 .4)])$.

\section{BRST}

5.1. Recall the definition of the BRST reduction due to Feigin (see [4]); the definition in the language of vertex algebras was given in [3, Section 4] see also [6, Appendix A]; for a more modern treatment see [1, 3.7], [2, 7.13].

Let $\mathfrak{a}$ be a finite-dimensional Lie algebra. Choose a base $\left\{a_{i}\right\}$ in $\mathfrak{a}$; denote the structure constants

$$
\left[a_{i}, a_{j}\right]=c_{p}^{i j} a_{p} .
$$

Recall that the Killing form $(\cdot, \cdot)_{(K)}: \mathfrak{a} \times \mathfrak{a} \rightarrow \mathbb{C}$ is given by

$$
\left(a_{i}, a_{j}\right)_{(K)}=c_{q}^{i p} c_{p}^{j q} .
$$

Let $\Pi \mathfrak{a}$ be the space $\mathfrak{a}$ with the reversed parity; denote by $\left\{\phi_{i}=\Pi a_{i}\right\}$ the corresponding base and by $\left\{\phi_{j}^{*}\right\}$ the dual base of $\Pi a^{*}$ given by

$$
\left\langle\phi_{i}, \phi_{j}^{*}\right\rangle=\delta_{i j}
$$

Let $C_{\mathrm{BRST}}(L \mathfrak{a})$ denote a graded vertex superalgebra generated by odd fields $\phi_{i}(z)$ of conformal dimension 1 and odd fields $\phi_{i}^{*}(z)$ of conformal dimension 0 with OPE

$$
\phi_{i}(z) \phi_{j}^{*}(w) \sim \frac{\delta_{i j}}{z-w} .
$$


We identify the spaces $\Pi \mathfrak{a}$ and $\Pi \mathfrak{a}^{*}$ with their obvious images in $C_{\mathrm{BRST}}(L \mathfrak{a})_{1}$ and $C_{\mathrm{BRST}}(L \mathfrak{a})_{0}$, respectively.

We introduce an odd element $D_{\mathfrak{a}} \in C_{\mathrm{BRST}}(L \mathfrak{a})$ of conformal dimension 1 by

$$
D_{\mathfrak{a}}=-\frac{1}{2} c_{p}^{i j} \phi_{p} \phi_{i}^{*} \phi_{j}^{*}
$$

Thus, we have the corresponding field $D_{\mathfrak{a}}(z)=\sum D_{\mathfrak{a} ; n} z^{-n-1}$ and we set

$$
d_{\mathfrak{a}}:=D_{\mathfrak{a} ; 0}
$$

The pair $\left(C_{\mathrm{BRST}}(L \mathfrak{a}), d_{\mathfrak{a}}\right)$ may be regarded as a chiral analogue of the Chevalley cochain complex $C(\mathfrak{a})$. However, in the chiral case the square $d_{\mathfrak{a}}^{2}$ may be nonzero. It is easy to compute it. Namely, we write down the OPE $D_{\mathfrak{a}}(z) D_{\mathfrak{a}}(w)$ using Wick theorem. We have

$$
D_{\mathfrak{a}}(z) D_{\mathfrak{a}}(w) \sim \frac{\left(a_{i}, a_{j}\right)_{(K)} \phi_{i}^{*}(z) \phi_{j}^{*}(w)}{(z-w)^{2}} .
$$

Therefore,

$$
d_{\mathfrak{a}}^{2}=\left(a_{i}, a_{j}\right)_{(K)} \int \phi_{i}^{*}(w)^{\prime} \phi_{j}(w) .
$$

COROLLARY 5.1. If the Lie algebra $\mathfrak{a}$ is nilpotent then $d_{\mathfrak{a}}^{2}=0$.

Indeed, the Killing form of a nilpotent Lie algebra is zero.

5.2. Let $(\cdot, \cdot): \mathfrak{a} \times \mathfrak{a} \rightarrow \mathbb{C}$ be an arbitrary symmetric invariant bilinear form ("level"). Recall that the vertex algebra $\mathscr{V}_{\mathfrak{a} ;(\cdot, \cdot)}$ is generated by even fields $a_{i}(z)$ of conformal weight 1 , subject to OPE

$$
a_{i}(z) a_{j}(w) \sim \frac{\left(a_{i}, a_{j}\right)}{(z-w)^{2}}+\frac{\left[a_{i}, a_{j}\right](w)}{z-w} .
$$

LEMMA 5.2. The rule

$$
a_{i} \longmapsto c_{q}^{i p} \phi_{q} \phi_{p}^{*}
$$

defines an embedding of vertex algebras

$$
\mathscr{V}_{\mathfrak{a} ;(\cdot, \cdot)_{\mathfrak{a} ;(K)}} \longrightarrow C_{\mathrm{BRST}}(L \mathfrak{a})
$$

5.3. Let $\mathcal{M}$ be a vertex module over $\mathscr{V}_{\mathfrak{a} ;-(\cdot, \cdot)_{\mathfrak{a} ;(K)}}$. We introduce a space

$$
C_{\mathrm{BRST}}(L \mathfrak{a} ; \mathcal{M}):=C_{\mathrm{BRST}}(L \mathfrak{a}) \otimes \mathcal{M} .
$$

According to Lemma 5.2, this space is canonically a graded (by conformal weight) $\mathscr{V}_{\mathfrak{a} ; 0}$-supermodule. This space is also graded by "fermionic charge"

$$
C_{\mathrm{BRST}}(L \mathfrak{a} ; M)=\oplus_{p \in \mathbb{Z}} C_{\mathrm{BRST}}^{p}(L \mathfrak{a} ; M),
$$

where we assign to $\phi_{i}$ (respectively, $\phi_{i}^{*}, m \in \mathcal{M}$ ) the charge -1 (respectively, 1,0 ). 
Introduce an odd element $D_{\mathfrak{a} ; \mu} \in C_{\mathrm{BRST}}(L \mathfrak{a} ; M)$ of conformal weight 1 and fermionic charge 1 by

$$
D_{\mathfrak{a} ; M}=\phi_{i}^{*} \otimes a_{i}+D_{\mathfrak{a}} \otimes 1
$$

It follows from (5.7) that

$$
D_{\mathfrak{a} ; M}(z) D_{\mathfrak{a} ; M}(w) \sim 0
$$

Therefore, setting

$$
d_{\mathfrak{a} ; \mu}:=\int D_{\mathfrak{a} ; \mu}(z)
$$

we get a differential

$$
d_{\mathfrak{a} ; \mu}^{2}=0
$$

By definition $d$ increases the fermionic charge by 1 .

The pair $\left(C_{\mathrm{BRST}}(L \mathfrak{a} ; M), d_{\mathfrak{a} ; \mathcal{M}}\right)$ is called the BRST complex of $L \mathfrak{a}$ with coefficients in $\mathcal{M}$, and its cohomology $H_{\mathrm{BRST}}^{*}(L \mathfrak{a} ; \mathcal{M})$ is called the BRST cohomology.

EXAMPLE 5.3. Let $N$ be a unipotent algebraic group with the Lie algebra $\mathfrak{n}$. Consider a $\mathscr{V}_{\mathfrak{n} ; 0}$-module $\mathscr{D}_{N ; 0}^{\mathrm{ch}}$ (note that according to Subsection 3.5 this algebra represents a unique isomorphism class of cdo's over $N$ ). Inside the loop algebra $L \mathfrak{n}=\mathfrak{n}\left[T, T^{-1}\right]$, consider two Lie subalgebras: $\mathfrak{n}_{-}$and $\mathfrak{n}_{+}$, generated by all elements $\tau T^{n}(\tau \in \mathfrak{n})$ with $n<0$ and $n \geq 0$, respectively. Then $\mathscr{D}_{N ; 0}^{\mathrm{ch}}$ is a free $\boldsymbol{n}_{-}$-module and a cofree (i.e., the dual module is free) $\mathfrak{n}_{+}$-module.

It follows that

$$
H_{\mathrm{BRST}}^{i}\left(L \mathfrak{n} ; \mathscr{D}_{N ; 0}^{\mathrm{ch}}\right)=0 \quad(i \neq 0) ; \quad H_{\mathrm{BRST}}^{0}\left(L \mathfrak{n} ; \mathscr{D}_{N ; 0}^{\mathrm{ch}}\right)=\mathbb{C},
$$

(cf. [6, page 178]).

\section{Homogeneous spaces}

6.1. Let $X$ be a smooth variety. We have an exact triangle

$$
\Omega_{X}^{[2,3\rangle} \rightarrow \Omega_{X}^{[2} \rightarrow C,
$$

where

$$
\Omega_{X}^{[2}: 0 \rightarrow \Omega_{X}^{2} \rightarrow \Omega_{X}^{3} \rightarrow \cdots
$$

$\left(\Omega_{X}^{2}\right.$ sitting in degree 0$)$ and

$$
C: 0 \rightarrow \frac{\Omega_{X}^{4}}{d \Omega_{X}^{3}} \rightarrow \Omega_{X}^{5} \rightarrow \cdots
$$

$\left(\Omega_{X}^{4} / d \Omega_{X}^{3}\right.$ sitting in degree 2). The following remark follows.

REMARK 6.1 (due to H. Esnault). The canonical map

$$
H^{i}\left(X ; \Omega_{X}^{[2,3\rangle}\right) \rightarrow H^{i}\left(X ; \Omega_{X}^{[2}\right)
$$

is injective for $i=2$ and bijective for $i=0,1$. 
6.2. Let $G$ be a simple algebraic group. In this section, we discuss the chiral differential operators on homogeneous spaces, $G / G^{\prime}$ where $G^{\prime}=N$-a unipotent subgroup, $G^{\prime}=P-$ a parabolic but not minimal parabolic, or $G^{\prime}=B-$ a Borel subgroup.

\section{THE CASE $G / N$}

6.3. Consider the projection $\pi: G \rightarrow X:=G / N$. The variety $X$ is quasiaffine, therefore we have

$$
H^{i}\left(X ; \Omega_{X}^{[2}\right)=H^{i} \Gamma\left(X ; \Omega_{X}^{[2}\right)=H_{\mathrm{DR}}^{i+2}(X) \quad(i \geq 1) .
$$

On the other hand,

$$
H_{\mathrm{DR}}^{*}(X)=H^{*}(X ; \mathbb{C})
$$

by Grothendieck's theorem, (cf. [9, Theorem 1']).

The projection $\pi$ is an affine morphism which is a Zariski locally trivial bundle with fiber $N$ isomorphic to an affine space, so $\pi^{*}: H^{*}(X ; \mathbb{C}) \stackrel{\sim}{\longrightarrow} H^{*}(G ; \mathbb{C})$. It follows that

$$
\pi^{*}: H^{i}\left(X ; \Omega_{X}^{[2}\right) \stackrel{\sim}{\longrightarrow} H^{i}\left(G ; \Omega_{G}^{[2}\right) \quad(i \geq 1) .
$$

We have a short exact sequence

$$
0 \longrightarrow \Theta_{G / X} \longrightarrow \Theta_{G} \longrightarrow \pi^{*} \Theta_{X} \longrightarrow 0
$$

and the vector bundles $\Theta_{G}, \Theta_{G / X}$ are trivial (a base of global sections of $\Theta_{G / X}$ is given by left invariant vector fields coming from the Lie algebra $\mathfrak{n}:=\operatorname{Lie}(N)$ ).

Therefore, we have

$$
\pi^{*} c\left(\mathscr{D i f f} \mathrm{ch}^{\mathrm{ch}}\right)=\pi^{*} \mathrm{ch}_{2}\left(\Theta_{X}\right)=\operatorname{ch}_{2}\left(\Theta_{G}\right)=0,
$$

hence

$$
c\left(\mathscr{D i f f} \mathrm{ch}^{\mathrm{ch}}\right)=0
$$

by Remark 6.1.

Therefore, we have the following corollary.

COROLLARY 6.2. The groupoid $\Gamma\left(X ; \mathscr{D i f f}_{X}^{\mathrm{ch}}\right)$ is nonempty. The set of isomorphism classes $\pi_{0}\left(\Gamma\left(X, D_{D i f f}^{\mathrm{ch}}\right)\right)$ is a torseur under $H_{\mathrm{DR}}^{3}(X)=H_{\mathrm{DR}}^{3}(G)$.

6.4. Let $\mathscr{D}_{G ;(\cdot, \cdot)}^{\mathrm{ch}}$ be the sheaf of chiral differential operators on $G$ of level $(\cdot, \cdot)$, where

$$
(\cdot, \cdot): \mathfrak{g} \times \mathfrak{g} \longrightarrow \mathbb{C}
$$

is a fixed symmetric invariant bilinear form on $\mathfrak{g}=\operatorname{Lie}(G)$, see Subsection 3.6. The form (6.11) is a scalar multiple of the Killing form

$$
(\cdot, \cdot)=c(\cdot, \cdot)_{\mathfrak{g} ;(K)}, \quad c \in \mathbb{C} .
$$

The Killing form on $\mathfrak{g}$ restricts to zero on $\mathfrak{n}$ (since the trace of a nilpotent endomorphism is zero). Therefore, we have the canonical embedding of vertex algebras

$$
\mathscr{V}_{\mathfrak{n} ; 0} \longleftrightarrow \mathscr{V}_{G ;(\cdot, \cdot)} \longleftrightarrow \mathscr{D}_{G ;(\cdot, \cdot)}^{\mathrm{ch}},
$$

so that the sheaf $\mathscr{D}_{G ;(\cdot, \cdot)}^{\mathrm{ch}}$ becomes a sheaf of $\mathscr{V}_{\mathfrak{n} ; 0}$-modules. 
Applying the BRST construction, see Subsection 5.3, to $\mathcal{M}=\pi_{*} \mathscr{D}_{G ;(\cdot, \cdot)}^{\mathrm{ch}}$ and $\mathfrak{a}=\mathfrak{n}$, we get a sheaf of BRST complexes $C_{\mathrm{BRST}}\left(L \mathfrak{n} ; \pi_{*} \mathscr{D}_{G ;(\cdot, \cdot)}^{\mathrm{ch}}\right)$ and BRST cohomology sheaves $H_{\mathrm{BRST}}^{*}\left(L \mathfrak{n} ; \pi_{*} \mathscr{D}_{G ;(\cdot, \cdot)}^{\mathrm{ch}}\right)$ over $X$. They are sheaves of $\mathbb{Z}_{\geq 0}$-graded vertex superalgebras.

THEOREM 6.3. We have

$$
H_{\mathrm{BRST}}^{i}\left(L \mathfrak{n} ; \pi_{*} \mathscr{D}_{G ;(\cdot, \cdot)}^{\mathrm{ch}}\right)=0 \quad(i \neq 0) .
$$

The sheaf $H_{\mathrm{BRST}}^{0}\left(L \mathfrak{n} ; \pi_{*} \mathscr{D}_{G ;(\cdot, \cdot)}^{\mathrm{ch}}\right)$ is an algebra of chiral differential operators over $X$.

The correspondence

$$
\mathscr{D}_{G ;(\cdot, \cdot)}^{\mathrm{ch}} \longmapsto H_{\mathrm{BRST}}^{0}\left(L \mathfrak{n} ; \pi_{*} \mathscr{D}_{G ;(\cdot, \cdot)}^{\mathrm{ch}}\right)
$$

induces a bijection of the sets of isomorphism classes

$$
\pi_{0}\left(\Gamma\left(G ; \mathscr{D i f f}{ }_{G}^{\mathrm{ch}}\right)\right) \stackrel{\sim}{\longrightarrow} \pi_{0}\left(\Gamma\left(X ; \text {Diff }_{X}^{\mathrm{ch}}\right)\right) .
$$

We use the notation $\mathscr{D}_{X ;(\cdot, \cdot)}^{\mathrm{ch}}$ for the cdo $H_{\mathrm{BRST}}^{0}\left(L \mathfrak{n} ; \pi_{*} \mathscr{D}_{G ;(\cdot, \cdot)}^{\mathrm{ch}}\right)$.

Note that higher direct images $R^{i} \pi_{*} \mathscr{D}_{G ;(\cdot, \cdot)}^{\mathrm{ch}}$ are trivial for $i>0$ since the morphism $\pi$ is affine and the sheaves $\mathscr{D}_{G ;(\cdot, \cdot)}^{\mathrm{ch}}$ admit a filtration whose quotients are coherent (in fact, locally free) $\mathrm{O}_{G}$-modules.

Proof (SKeTCH). Locally on $X$ the projection $\pi: G \rightarrow X$ is isomorphic to the direct product $U \times N \rightarrow U$. If $\mathscr{D}$ is an algebra of chiral differential operators on $U \times N$ then $\mathscr{D} \stackrel{\sim}{\longrightarrow} \mathscr{D}_{U} \otimes \mathscr{D}_{N}$, where $\mathscr{D}_{U}$ (respectively, $\mathscr{D}_{N}$ ) is an algebra of differential operators on $U$ (respectively, $N$ ). Now the first claim of the theorem follows from (5.18).

The second claim is Corollary 6.2.

COROLlary 6.4. The dual embedding

$$
j_{R}: \mathscr{V}_{G ;(\cdot, \cdot)^{o}} \longrightarrow \mathscr{D}_{G ;(\cdot, \cdot)}^{\mathrm{ch}}
$$

defined in Corollary 4.2 induces a canonical morphism of vertex algebras

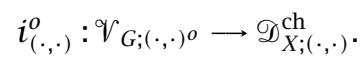

Indeed, we know that (6.17) commutes with the left action of $\hat{\mathfrak{g}}$ hence with the BRST differential.

In particular, the Kac-Moody algebra $\hat{\mathfrak{g}}$ at level $(\cdot, \cdot)^{o}$ acts canonically on Wakimoto modules which may be defined as the spaces of sections $\Gamma\left(U ; \mathscr{D}_{X,(\cdot, \cdot)}^{\mathrm{ch}}\right)$, where $U$ is a big cell. This is a result due to Feigin-Frenkel obtained in $[5,6,7]$ in a different way.

The CASE $G / B$

6.5. Let $B \subset G$ be a Borel subgroup, $\pi: G \rightarrow X:=G / B$. Then $X$ is a smooth projective variety and we have

$$
\begin{gathered}
H^{p}\left(X ; \Omega_{X}^{q}\right)=0 \quad(p \neq q), \\
H^{i}\left(X ; \Omega_{X}^{i}\right)=H^{2 i}(X ; \mathbb{C}) .
\end{gathered}
$$


It follows that $H^{2}\left(X ; \Omega^{[2}\right)=H^{4}(X ; \mathbb{C})$, and Theorem 7.5 in [8] says that the image of $c\left(\mathscr{D i f f}{ }_{X}^{\mathrm{ch}}\right)$ in $H^{4}(X ; \mathbb{C})$ is equal to

$$
2 \operatorname{ch}_{2}\left(\Theta_{X}\right):=c_{1}^{2}\left(\Theta_{X}\right)-c_{2}\left(\Theta_{X}\right),
$$

where $c_{i}\left(\Theta_{X}\right) \in H^{2 i}(X ; \mathbb{C})$ are the Chern classes of the tangent bundle $\Theta_{X}$.

LEMMA 6.5. We have

$$
\operatorname{ch}_{2}\left(\Theta_{X}\right)=0 .
$$

Proof. The space $H^{2}(X ; \mathbb{C})$ may be identified with the complexification of the root lattice of $G$. The classical theorem by J. Leray says that the cohomology algebra $H^{*}(X ; \mathbb{C})$ is equal to the quotient of the symmetric algebra of the space $H^{2}(X ; \mathbb{C})$ modulo the ideal generated by the subspace of invariants of the Weyl group $W$ having positive degree (cf. [10, Théoreme 2.1b]).

The class $\left[\Theta_{X}\right]$ in the Grothendieck group of vector bundles is equal to the sum $\sum\left[\mathscr{L}_{\alpha}\right]$, where $\alpha$ runs through all negative roots, and $\mathscr{L}_{\alpha}$ is the line bundle with $c_{1}\left(\mathscr{L}_{\alpha}\right)=\alpha$. Hence $\operatorname{ch}_{2}\left(\Theta_{X}\right)=\sum \alpha^{2}$; this element is invariant under the action of $W$, therefore its image in $H^{4}(X ; \mathbb{C})$ is zero.

This lemma implies that

$$
c\left(\mathscr{D i f f}_{X}^{\mathrm{ch}}\right)=0
$$

by Remark 6.1. On the other hand, it follows from Remark 6.1 and (6.19) that

$$
H^{i}\left(X ; \Omega_{X}^{[2,3\rangle}\right)=0 \quad(i=0,1) .
$$

Thus, by [8], we get the following theorem.

THEOREM 6.6. The groupoid $\Gamma\left(X ; D_{i f f}^{\mathrm{ch}}\right)$ is nonempty and trivial. In other words, there exists a unique, up to a unique isomorphism, algebra of chiral differential operators $\mathscr{D}_{X}^{\mathrm{ch}}$ over $X$.

6.6. We construct the algebra $\mathscr{D}_{X}^{\text {ch }}$ using the BRST reduction. Consider the sheaf $\mathscr{D}_{G ;(\cdot,)}^{\mathrm{ch}}$ as in Subsection 6.4. Let $(\cdot, \cdot)_{\mathfrak{6}}$ denote the restriction of $(\cdot, \cdot)$ to $6:=\operatorname{Lie}(B)$.

We have a canonical embedding of vertex algebras

$$
\mathscr{V}_{\mathfrak{b} ;(\cdot, \cdot)_{\mathfrak{b}}} \longrightarrow \mathscr{D}_{G ;(\cdot, \cdot)}^{\mathrm{ch}},\left.\quad(\cdot, \cdot)_{\mathfrak{g} ;(K)}\right|_{\mathfrak{b} \times \mathfrak{b}}=2(\cdot, \cdot)_{\mathfrak{b} ;(K)} .
$$

Therefore, to get the minus Killing on $\mathfrak{b}$ we have to start from $-(\cdot, \cdot)_{\mathfrak{g},(K)} / 2$, that is, from the critical level on $\mathfrak{g}$. Thus, by construction 5.3 , we have the following.

6.6.1. The BRST complex $C_{\mathrm{BRST}}\left(L \mathfrak{b} ; \pi_{*} \mathscr{D}_{G ;(\cdot, \cdot)}^{\mathrm{ch}}\right)$ is defined if and only if $(\cdot, \cdot)=(\cdot, \cdot)_{\mathfrak{g} ; \text { crit }}$, that is, on the critical level, see (4.5).

We denote the algebra $\mathscr{D}_{G ;(\cdot, \cdot))_{\text {g; } ; \text { rit }}}^{\mathrm{ch}}$ by $\mathscr{D}_{G ; \text { crit }}^{\mathrm{ch}}$.

6.7. Let $\mathscr{V}_{6 ; \text { crit }}$ denote the vacuum module on the critical level $\mathscr{V}_{6 ;(\cdot, \cdot))_{6 ; \text { crit }}}$. Let $\mathcal{M}$ be a vertex algebra equipped with a morphism of vertex algebras $\mathscr{V}_{6 ; \text { crit }} \rightarrow \mathcal{M}$.

We have a decomposition

$$
\mathfrak{b}=\mathfrak{h} \oplus \mathfrak{n},
$$


where $\mathfrak{n} \subset \mathfrak{b}$ (respectively, $\mathfrak{h} \subset \mathfrak{b}$ ) is the maximal nilpotent (respectively, the Cartan) subalgebra; $\mathfrak{n}$ is spanned by elements $e_{\alpha}, \alpha$ being a positive root. We define a vertex subalgebra

$$
C_{\mathrm{BRST}}(L \mathfrak{b}, \mathfrak{h} ; \mathcal{M}) \subset C_{\mathrm{BRST}}(L \mathfrak{b} ; \mathcal{M}) .
$$

The vector space $C_{\mathrm{BRST}}(L 6 ; M)$ is spanned by all the monomials

$$
h_{1 ; i_{1}} \cdots \cdots h_{1 ; j_{1}}^{*} \cdots \cdots e_{\alpha_{1} ; k_{1}} \cdots e_{\alpha_{1}^{\prime} ; l_{1}}^{*} \cdots \otimes m_{\mu},
$$

where the indices $i_{p}, j_{p}, k_{p}, l_{p}$ denote the conformal weight, $i_{p}, k_{p} \geq 1 ; j_{p}, l_{p} \geq 0$, $m_{\mu} \in \mathcal{M}$ is a vector of weight $\mu \in \mathfrak{h}^{*}$.

By definition, the subspace $C_{\mathrm{BRST}}(L \mathfrak{G}, \mathfrak{h} ; \mathcal{M})$ is spanned by all monomials (6.28) such that

(a) all $j_{p} \geq 1$;

(b) $\sum \alpha_{p}-\sum \alpha_{q}^{\prime}+\mu=0$.

It is a vertex subalgebra. One checks that the BRST differential $d$ in $C_{\mathrm{BRST}}(L \mathfrak{b} ; M)$ preserves $C_{\mathrm{BRST}}(L \mathfrak{G}, \mathfrak{h} ; \mathcal{M})$.

We define the relative BRST cohomology $H_{\mathrm{BRST}}^{*}(L \mathfrak{G}, \mathfrak{h} ; M)$ as the cohomology of $C_{\mathrm{BRST}}(L \mathfrak{b}, \mathfrak{h} ; M)$ with respect to this differential. It is canonically a vertex algebra.

6.8. Applying the previous definition to $M=\pi_{*} \mathscr{D}_{G ; \text { crit }}^{\text {ch }}$, we get the BRST cohomology sheaves $H_{\mathrm{BRST}}^{*}\left(L \mathfrak{G}, \mathfrak{h} ; \pi_{*} \mathscr{D}_{G \text {; crit }}^{\mathrm{ch}}\right)$ on $X$.

THEOREM 6.7. We have

$$
H_{\mathrm{BRST}}^{i}\left(L \mathfrak{b}, \mathfrak{h} ; \pi_{*} \mathscr{D}_{G ; \mathrm{crit}}^{\mathrm{ch}}\right)=0 \quad(i \neq 0),
$$

and the sheaf of vertex algebras $H_{\mathrm{BRST}}^{0}\left(L \mathfrak{b}, \mathfrak{h} ; \pi_{*} \mathscr{D}_{G ; \text { crit }}^{\mathrm{ch}}\right)$ is canonically isomorphic to $\mathscr{D}_{X}^{\mathrm{ch}}$.

The proof is the same as that of Theorem 6.3.

COROLlARY 6.8. We have a canonical isomorphism of vertex algebras

$$
H^{*}\left(X ; \mathscr{D}_{X}^{\mathrm{ch}}\right)=H_{\mathrm{BRST}}^{*}\left(L \mathfrak{L}, \mathfrak{h} ; \Gamma\left(G ; \mathscr{D}_{G ; \text { crit }}^{\mathrm{ch}}\right)\right) .
$$

Indeed, as we have already remarked, sheaves of cdo $\mathscr{D}_{G}^{\text {ch }}$ on $G$ have a filtration whose graded quotients are vector bundles; but the variety $G$ and the morphism $\pi$ are affine, hence $H^{i}\left(G ; \mathscr{D}_{G}^{\mathrm{ch}}\right)=R^{i} \pi_{*} \mathscr{D}_{G}^{\mathrm{ch}}=0$ for $i>0$.

COROLLARY 6.9. The dual embedding

$$
j_{R}: \mathscr{V}_{G ; \text { crit }} \longrightarrow \mathscr{D}_{G \text {;crit }}^{\mathrm{ch}}
$$

defined in Corollary 4.2 induces a canonical morphism of vertex algebras

$$
i_{\text {crit }}: \mathscr{V}_{G ; \text { crit }} \longrightarrow \mathscr{D}_{X}^{\text {ch }}
$$

Indeed, we know that (6.31) commutes with the left action of $\hat{\mathfrak{g}}$ hence with the BRST differential. 
In particular, the Kac-Moody algebra $\hat{\mathfrak{g}}$ on the critical level acts canonically on Wakimoto modules which may be defined as the spaces of sections $\Gamma\left(U ; \mathscr{D}_{X}^{\mathrm{ch}}\right)$, where $U$ is a big cell. This is a result of Feigin-Frenkel proved in $[5,6,7]$ in a different way.

THE CASE $G / P$

6.9. Let $P \subset G$ be a parabolic but not Borel, $\mathfrak{p}=\operatorname{Lie}(P), \pi: G \rightarrow X:=G / P$. The discussion in Subsection 6.5 applies as it is, except for Lemma 6.5, which is replaced by the following lemma.

LEMMA 6.10. We have

$$
\operatorname{ch}_{2}\left(\Theta_{X}\right) \neq 0 .
$$

Hence $c\left(\mathscr{D i f f} \mathrm{ch}_{X}^{\mathrm{h}}\right) \neq 0$, and we get the following theorem.

THEOREM 6.11. The groupoid $\Gamma\left(X ; D_{\mathrm{iff}_{X}^{\mathrm{ch}}}\right)$ is empty. In other words, there is no cdo over $X$.

6.10. We see how this fact is reflected in the BRST world. We would like to get a sheaf of cdo over $X$ as the BRST cohomology of $L p$ with coefficients in some sheaf $\pi_{*} \mathscr{D}_{G ;(\cdot, \cdot)}^{\mathrm{ch}}$. However, no form $(\cdot, \cdot)$ on $G$ restricts to the Killing form on $\mathfrak{p}$, which implies that the square of the BRST differential in $C_{\mathrm{BRST}}\left(L \mathfrak{p} ; \pi_{*} \mathscr{D}_{G ;((\cdot,))}^{\mathrm{ch}}\right)$ is always nonzero. Thus, the BRST cohomology is not defined, which is compatible with Theorem 6.11.

ACKNOWLEDGEMENTS. This work was done while the authors were visiting Institut des Hautes Études Scientifiques in Bures-sur-Yvette and Max-Planck-Institut für Mathematik in Bonn. We are grateful to these institutes for the hospitality.

Fyodor Malikov is partially supported by NSF grant.

\section{REFERENCES}

[1] A. Beilinson and V. Drinfeld, Chiral algebras, preprint.

[2] Quantization of Hitchin integrable system and Hecke eigensheaves, preprint.

[3] B. Feigin and E. Frenkel, Affine Kac-Moody algebras at the critical level and Gel'fand-Dikir algebras, Infinite Analysis, Part A, B (Kyoto, 1991), Adv. Ser. Math. Phys., vol. 16, World Sci. Publishing, New Jersey, 1992, pp. 197-215. MR 93j:17049.

[4] B. L. Feigin, Semi-infinite homology of Kac-Moody and Virasoro Lie algebras, Uspekhi Mat. Nauk 39 (1984), no. 2(236), 195-196 (Russian). MR 85g:17003. Zbl 544.17009.

[5] B. L. Feigin and E. V. Frenkel, A family of representations of affine Lie algebras, Uspekhi Mat. Nauk 43 (1988), no. 5(263), 227-228 (Russian). MR 89k:17016. Zbl 657.17013.

[6] _ Affine Kac-Moody algebras and semi-infinite flag manifolds, Comm. Math. Phys. 128 (1990), no. 1, 161-189. MR 92f:17026. Zbl 722.17019.

[7] _ Representations of affine Kac-Moody algebras and bosonization, Physics and Mathematics of Strings, World Sci. Publishing, New Jersey, 1990, pp. 271-316. MR 92d:17025. Zbl 734.17011.

[8] V. Gorbounov, F. Malikov, and V. Schechtman, Gerbes of chiral differential operators, Math. Res. Lett. 7 (2000), no. 1, 55-66, http://arxiv.org/abs/math.AG/0003170. CMP 1748 287. Zbl 991.40966.

[9] A. Grothendieck, On the de Rham cohomology of algebraic varieties, Inst. Hautes Études Sci. Publ. Math. (1966), no. 29, 351-359. MR 33\#7343. Zbl 145.17602.

[10] J. Leray, Sur l'homologie des groupes de Lie, des espaces homogènes et des espaces fibrés principaux, Colloque de Topologie (Espace Fibrés), Bruxelles, 1950, Georges 
Thone, Liège; Masson et Cie., Paris, 1951, pp. 101-115 (French). MR 12,802f. Zbl 042.41801.

[11] F. Malikov, V. Schechtman, and A. Vaintrob, Chiral de Rham complex, Comm. Math. Phys. 204 (1999), no. 2, 439-473. MR 2000j:17035a. Zbl 952.14013.

VASSILY Gorbounov: Department of MATHEMATICS, UniVERSITY OF KENTUCKY, LEXINGTON, KY 40506, USA

E-mail address: vgorb@ms. uky. edu

Fyodor Malikov: DePartment of MATHEMATICS, UniVERSITY OF SOUTHERN CALIFORNiA, LOS ANGELES, CA 90089, USA

E-mail address: fma7ikov@mathj.usc.edu

VADIM SCHECHTMAN: LABORATOIRE EMILE PICARD, UFR MIG, UNIVERSITÉ PAUl SABATIER, 118 Route DE NARBonNe, 31062 TOUlOUSE CEDEX 4, FranCE

E-mail address: schechtman@picard.ups-t7se.fr 


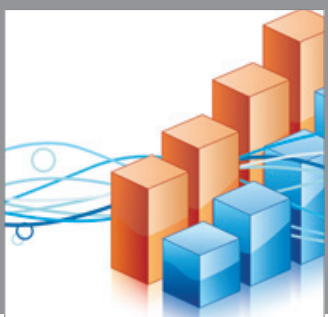

Advances in

Operations Research

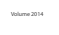

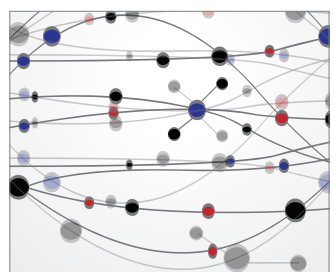

\section{The Scientific} World Journal
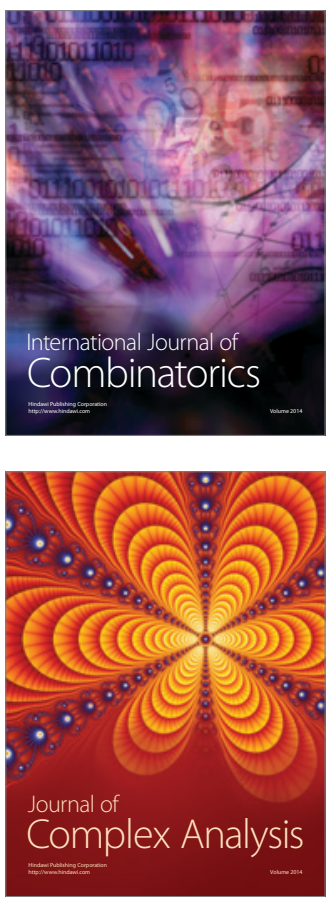

International Journal of

Mathematics and

Mathematical

Sciences
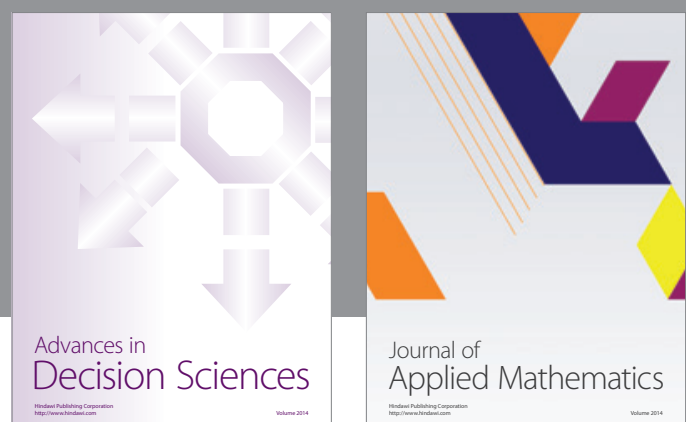

Journal of

Applied Mathematics
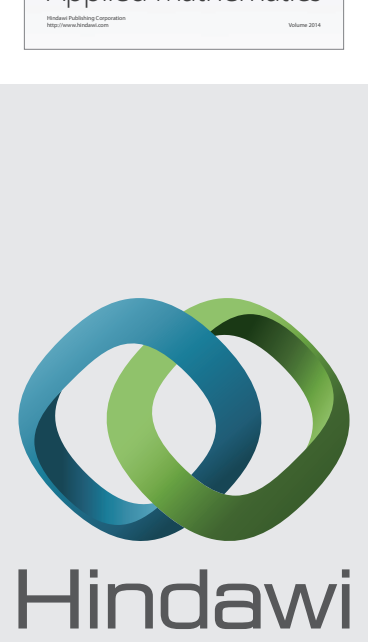

Submit your manuscripts at http://www.hindawi.com
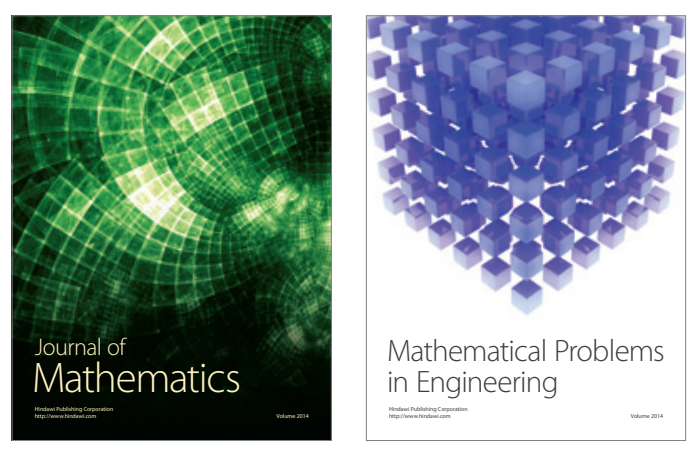

Mathematical Problems in Engineering
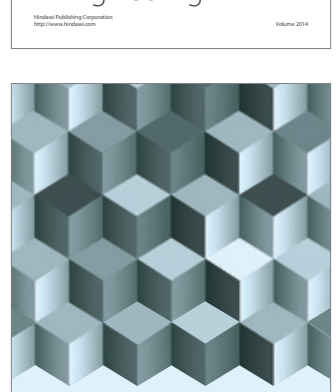

Journal of

Function Spaces
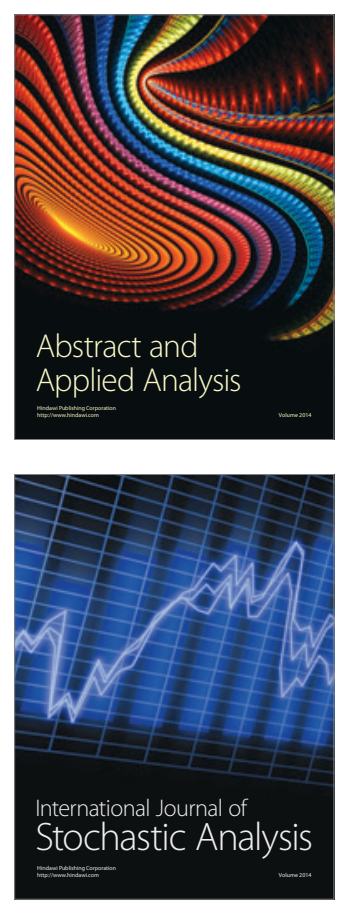

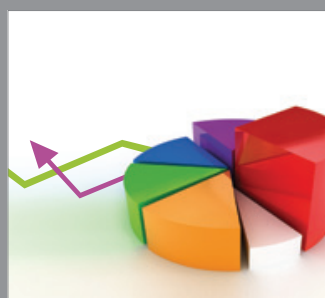

ournal of

Probability and Statistics

Promensencen
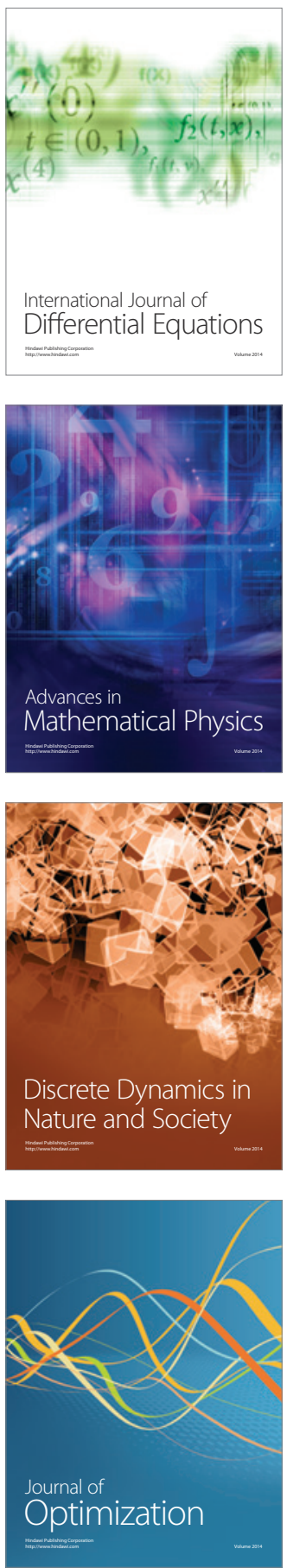